\title{
Contribution à la faune malacologique de Madagascar.
}

\author{
Par \\ NILS HJ. ODHNER, \\ Stockholm.
}

Aver 4 planches et 4 figures dans le texte.

Communiqué le 9 Octobre 1918 par HJ. Théel et E. Lönnberg.

En 1911-12, pendant une expédition scientifique à Madagascar, M. W. KaUdern de Stockholm avait l'occasion de recueillir, dans diverses localitées, plusieurs espèces de mollusques. Comme la faune malacologique de Madagascar est encore loin d'être suffisamment étudiée et comme les collections faites contiennent beaucoup de spécimens intéressants, je les ai considerées comme méritant une publication spéciale utile à la science. Les collections comprennent pour la plupart des mollusques marins mais aussi quelques formes terrestres et aquatiques. Quant à la première catégorie, plusieurs de ces espèces sont nouvelles pour la science ou pour la faune malgache. Une grande quantité des mollusques marins a été recueillie, il est vrai, à marée basse sur les rochers de la côte ou sur des récifs de coraux; ces formes, par cela, ont moins de valeur. Mais une autre partie des formes, au contraire, sont obtenues par dragage sur la côte. Le résultat des dragages a été assez riche, d'autant plus que cette méthode d'exploration n'a pas auparavant été employée aux localités respectives. Malbeureusement, deux dragages seulement furent effectuées, l'un à Tamatave et l'autre à 
Majunga. Celui-là fut fait en deça du récif de coraux, à 20-25 mètres de profondeur, fond sableux, le 2 mars 1912. A Majunga le dragage fut entrepris plus tard en mars, dans le canal profond de la Baie de Bombetoke à 50-60 mètres; le fond était composé de feuilles et de débris de plantes, transportés par le fleuve Betsiboka.

Les collections de mollusques marins comprennent en tout 200 espèces, sur lesquelles le nombre des formes décrites dans ce mémoire comme nouvelles monte à 16 . Un résultat des plus intéressants est la découverte de l'occurrence sur la côte de Madagascar d'une espèce de Choneplax, genre qui était connu jusqu'ici seulement dans les Indes occidentales. Outre les espèces nouvelles il y a aussi dans les collections un nombre de formes insuffisamment connues qui sont maintenant introduites dans la faune de Madagascar, par ex. Chione siamensis Lynge et Terebra polygonia Reeve.

Parmi les mollusques terrestres deux espèces du genre Vaginula offrent un intérêt spécial. Elles ont été capturées à Tamatave et appartiennent à un groupe nouveau du genre Eleutherocaulis Simroth, auquel je propos le nom de Vallicaulis. L'une des espèces est nouvelle et l'autre n'a été que très insuffisamment étudiée.

Dans une des grottes funéraires aux environs de Catsèpe quelques espèces de mollusques terrestres furent recueillies à l'état de fossile, dont trois n'ont pas été auparavant décrites.

Outre les collections faites par M. KAUDERN, je rapporte aússi quelques espèces de Tuléar, recueillies par M. K. AFzelıus de Stockholm, qui séjournait à Madagascar en même temps que M. KAUDERN pour des explorations botaniques.

Les collections décrites dans ce mémoire appartiennent au Musée d'histoire naturelle (Riksmuseum) à Stockholm.

C'est un plaisir pour moi d'exprimer ici ma gratitude à M. le Professeur A. VAyssik̀ne à Marseille qui a bien voulu parcourir mon manuscrit et vérifier mes déterminations.

\section{Mollusques marins de la côte du nord-ouest.}

Ces collections sont faites pour la plupart dans les environs de Majunga (Amborovy, Catsèpe) et à l'île de Mahakamby au large de la côte. 
Pinna nigra Chemnitz. Majunga, 1 valve gauche, long. $95 \mathrm{~mm}$, courbée, épineuse vers le bord. - Amborovy, 1 ex., $100 \mathrm{~mm}$, épineux vers le bord.

Pinna bicolor Chemnitz, var. Pl. 1, fig. 1. Mahakamby, aug. 1912, 3 ex., max. long. $192 \mathrm{~mm}$. Par leur extrémité postérieure abruptement tronquée et par leur forme élancée, les présents exemplaires correspondent à $P$. fumata et à l'espèce voisine $P$. euglypta, tandis qu'elles diffèrent de $P$. bicolor, par l'extrémité postérieure doucement arrondie de cette espèce. Mais $P$. bicolor et $P$. fumata n'ont pas une sculpture si prononcée que dans l'espèce présente; des cordons radiants, bas, étroits, traversés de sillons de squamelles en forme de rebords profondement recourbés en arrière. Ce n'est que vers le borả postérieur que ces plis se lèvent en forme de squamelles véritables, quoique très courtes. Une sculpture semblable est caractéristique de $P$. regia Hanley (Reeve, Conch. Icon. 11, fig. 56), mais la couleur de cette espèce consiste en zones transversales obscures, tandis que chez l'espèce présente un dessin semblable se montre seulement au milieu, passant postérieurement en un dessin rayonnant comme chez $P$. bicolor. La couleur, cependant, semble varier, puisque certains exemplaires ont leurs rubans rayonnants prédominant aussi sur la moitié antérieure. En tous cas, la coloration rayonnante est prédominante sur la transversale, et la détermination, par ce fait, doit valoir, d'autant plus que des exemplaires de $P$. bicolor au musée de Stockholm (R. M.) montrent une sculpture presque également forte et marquée et portent de courtes squamelles vers le bord postérieur. La forme présente est probablement à considérer comme une variété de $P$. bicolor. Elle correspond entièrement à des exemplaires du nord-ouest de l'Australie, récoltés par M. MJöBERG. ${ }^{1}$

Pecten porphyrius Chemnitz. Majunga, dragage, 4 valves, max. diam. $17 \mathrm{~mm}$. Ces exemplaires ressemblent à $P$. senatorius ReEve (Conch. Icon. 8, fig. 81), qui est, selon v. MaRTens (1880), ${ }^{2}$ identique à cette espèce de Chemnitz.

${ }^{1}$ N. Odhner, Results of Dr. E. MJöberg's Swedish Scientific Expeditions to Australia 1910-13. Mollusca. K. Sv. Vet. Akad. Handl. Bd. 52. Stockholm 1917.

¿ Beiträge zur Meeresfauna der Insel Mauritius und der Seychellen. 
Plicalula imbricata Menke. Majunga, la plage, 1 ex., long. $23 \mathrm{~mm}$.

$P$. australis Lamarck. Majunga, dragage, 2 valves, max. long. $17 \mathrm{~mm}$. Quoique usées, elles présentent distinctement la seulpture caractéristique, le plissement ramifié et les taches éparses qui donnent une similitude entière avec la fig. 10 (Conch. Icon. 19) de ReEve. Vers le bord on aperçoit aussi des traces d'épines (Reeve fig. $10 \mathrm{e}$ ). Cette espèce n'est pas mentionnée par v. Martens (1880), mais cet auteur porte sur sa liste la $P$. multiplicata la plus approchante, qui n'a pas de taches mais un dessin en lignes.

Pteria (Meleagrina) vulgaris Schumacher. Majunga, 4 valves, max. diam. (hauteur) $35 \mathrm{~mm}$. Elles correspondent à des exemplaires du Golfe Persique ainsi qu'à ceux de PortNatal (R. M., Avicula occa R enve, qui est synonyme de l'espèce présente; comparer LYNGE 1909). ${ }^{1}$ La coloration est minutieusement formée de fines lignes rouges concentriques en zigzag; outre cela se trouvent typiquement de larges rayons radiants.

Vulsella attenuata ReEve ( $V$. spongiarum ReEve, non LAmarck). Majunga, 2 ex. dans des éponges, max. long. $27 \mathrm{~mm}$. Ces exemplaires diffèrent un peu de la forme typique par leurs crochets plus fortement divergents et leur septum ligamentaire moins profondement descendant. Pour le nomenclature je réfère à E. A. Sмiтh (1911). ${ }^{2}$

Malleus regula Fonskål. Majunga, dragage, plusieurs ex., max. long. $22 \mathrm{~mm}$, complètement ressemblant à des ex. de Suez (M'ANDREw). - Var. decurtata, sur la rivière, 2 ex., max. long. $14 \mathrm{~mm}$.

Perna vulsella Lamarck. Mahakamby ( ${ }^{26} / 8$ 1912), 3 ex., max. long. $45 \mathrm{~mm}$. Malgré leur couleur entièrement jauneblanche, ces coquilles appartiennent bien à l'espèce présente. Elles sont sculptées de petites côtes indistinctes à direction longitudinale, interrompues ça et là, et elles sont plus épaisses que chez le type et de plus inéquivalves, puisque la valve gauche est plus fortement convexe qu'en général. Des formes

1 Marine Lamellibranchiata. The Danish Exper. to Siam 1899-1900. Mém. de l'Acad. des Sci. et des Lettres de Danernark. Copenhague.

${ }^{2}$ On the recent species of the Genus Vulsella 1'roc. Malac. Soc. 9. 
intermédiaires reliant cette variété au type à couleur radiante, se trouvent à R. M. de Keeling Island, Philippines, Sumatra, Tahiti, Honolulu, et des Indes occidentales. Clessin (Martini \& Chemnitz, Conch. Cab.) a cité l'espèce de l'Afrique occidentale.

P. isognomon Linné. Mahakamby, aug. 1912, plusieurs ex., max. long. $120 \mathrm{~mm}$. Très variable en forme: partie inférieure parfois fléchie perpendiculairement à la partie supérieure et se pliant à droite, de manière que la valve gauche devient convexe, la droite concave; oreillette postérieure très courte (ou assez longue dans certains échantillons); les sillons ligamentaires varient en nombre d'après la longueur du bord de la charnière (16 observés comme max.).

Anomia sol Reeve. Mahakamby, 1 ex., diam. 19,5 mm, attaché sur Pinna bicolor. Valve supérieure grise-blanche, un peu brunâtre, irrégulièrement bossuée, avec des côtes radiantes très serrées, visibles surtout à la partie postérieure et couvertes de séries de petits nodules; surface sculptée de nombreuses et fines lignes microscopiques d'une course irrégulière. Valve inférieure plate ou faiblement concave, presque lisse, excepté des lignes d'accroissement en forme de lamelles.

Ostrea vitrefacta Sowerby. Mahakamby, sur Pinna bicolor, 1 ex., long. $21 \mathrm{~mm}$.

O. radiata Valenciennes. Mahakamby, sur Murex ramosus, 2 petites valves supérieures, facilement reconnaissables par les denticules le long du bord.

O. hyotis Linné. Mahakamby, aug. 1912, 2 valves gauches, max. long. ca. $140 \mathrm{~mm}$. - Majunga, la plage, 1 ex., long. $40 \mathrm{~mm}$; dragage, quelques petits ex.

o. cucullina Deshayes. Majunga, la plage, 3 ex., max. long. $24 \mathrm{~mm}$. La taille de ces exemplaires est plus irrégulière que la fig. $7, \mathrm{Pl}$. XXXII, chez Deshayes (1863), ${ }^{1}$ mais la valve supérieure est plane et l'autre est profondement concave comme typiquement. La couleur, aussi, est le brun caractéristique que représente la figure. Selon Dhshayes la forme est assez variable.

${ }^{1}$ Conchyliologio de l'île de la Réunion. Paris. 
Septifer bilocularis Linné. Majunga, la plage, 2 ex., long: $35 \mathrm{~mm}$; dragage, quelques fragments et coquilles. - Mahakamby $\left({ }^{26} / 8\right), 4$ ex., max. long. $33,5 \mathrm{~mm}$, couleur à l'intérieur bleue, à l'extérieur verte foncée tirant sur le jaune.

Modiola philippinarum Hanley. Amborovy (28/8), 1 ex., long. $74 \mathrm{~mm}$.

Modiolaria difficilis Deshayes. Majunga, dragage, 1 valve gauche et un fragment dextre; celle-là ressemble en forme et en sculpture aux figs. 22-24 (Pl. 3) de Deshayes (1863). Comme le bord ventral est sinueux au centre, la coquille est indistinctement carénée, la carène divisant la striation en deux parties rayonnantes l'une vers le bord ventral, l'autre en arrière. Une zone médiane lisse s'étend des crochets; devant cette zone apparaissent de nouveau des stries radiantes, mais qui ne sont pas mentionnées ni figurées par Deshayes. Malgré ce fait et bien que la forme diffère un peu par l'absence de l'angle entre les bords supérieurs et postérieurs, l'identité est vraisemblable si l'on considère la forte variation sculpturelle et formelle chez d'autres espèces de Modiolaria (par exemple $M$. discors). $M$. africana, décrite par Bartsch 1915, ${ }^{1}$ d'une couleur jaune pâle, est sans doute la même espèce. Les dimensions de l'ex. présent sont: longueur $13 \mathrm{~mm}$, hauteur $6 \mathrm{~mm}$, épaisseur d'une valve 4,2 $\mathrm{mm}$.

Arca antiquata Linné, var. scapha Meuschen. Majunga, la plage, 1 valve gauche, long. $30 \mathrm{~mm}$, ressemblant à des ex de la mer Rouge au Musée de Stockholm (R. M.).

A. natalensis Krauss. Majunga, la plage, 3 valves gauches, max. long. $34 \mathrm{~mm}$. Je suis convaincu qu'elles appartiennent à cette espèce après une comparaison faite avec quelquesuns des types de Krauss conservés au musée; la forme et la sculpture sont identiques (celle-là consiste en 30 côtes lisses, aplaties; les interstices portent des stries serrées). Distribution: Mauritius (v. Martens 1880).

A. lactea Linné. Majunga, dragage, 1 valve gauche + 2 valves droites, max. long. $13 \mathrm{~mm}$. Ces ex. concordent d'un

${ }^{1}$ Rep on ... S. African Marine Mollusks. Smithsonian Inst. Bull. 91. Washington. 
côté avec $A$. striata Reeve de Suez (coll. M'Andrew), et de l'autre avec $A$. lactea de Port Natal (orig. Krauss 1848), ${ }^{1}$ et ils diffèrent par leur sculpture plus fine de l'A. solida Sow., que M. Lamy (1907) considère comme une variété de l'A. afra.

A. ehrenbergi Dunker. Majunga, la plage, 1 v. dr., long. $53 \mathrm{~mm}$, ressemblant en forme et en sculpture à la fig. de Kobelt (Martini \& Chemnitz 1891, pl. 25, fig. 5, 6). Selon КовеLт (1891) et Lamy (1907) l'espèce se trouve dans la mer Rouge.

A. navicularis Bruguik̀re. Majunga, la plage, 1 v. g. +1 v. dr. de deux ex., max. long. $40 \mathrm{~mm}$. Comparés et concordants avec des exemplaires du Japon, et avec la fig. de KовеLт (1891, pl. 4, fig. 7).

A. plicata Chemnitz (= divaricata Sowerby). Majunga, plusieurs ex., max. long. $20 \mathrm{~mm}$. Pour la synonymie et la distribution voir LYNGE 1909.

A. complanata Chemnitz. Majunga, la plage, 2 ex., max. long. $59 \mathrm{~mm}$; dragage, $7 \mathrm{ex}$., max. long. $22 \mathrm{~mm}$. - Mahakamby ( $26 / \mathrm{s}$ 1912), 2 ex., max. long. $28,5 \mathrm{~mm}$. - L'un de ces ex. premiers se lie à l'Arca nivea Chemnitz (voir Reeve, Conch. Icon. 2, Arca, sp. 96) par sa carène largement arrondie s'étendant des crochets au coin postéro-inférieur. L'exemplaire plus petit, au contraire, n'a pas cette formation et s'accorde avec des formes de l'A. decussata (= complanata) res Philippines et des îles Pacifiques (par ex. Eimeo, R. M.). Il est, en outre, complètement semblable à $A$. nivea Krauss (types à R. M.). C'est alors très vraisemblable que l'A. nivea n'est à considérer que comme une forme tuméfiée de l' $A$. complańata. Dunker (Novitates p. 124, pl. 41, var.) ainsi que Kовецт 1891 (Conch. Cab.) s'accordent dans l'opinion que l'A. nivea n'est pas à séparer de l'A. velata; cette forme est considérée par Lynge (1909) comme synonyme de l'A. complanata et cet auteur ajoute que l'A. nivea n'est pas "sufficiently defined as a species, and it needs revision». Bien que je n'ai pas vu d'exemplaire typique de l'A. nivea;

${ }^{1}$ Die südafrikanischen Mollusken. Stuttgart. 
il me semble, cependant, que la forme de Madagascar indique qu'elle doit être regardée comme synonyme de l'espèce présente.

A. imbricata Bruguière, var. avellana LAmarck. Mahakamby ( $26 / 8$ 1912), 2 ex., max. long. $26,5 \mathrm{~mm}$.

A. lateralis Reeve. Majunga, dragage, plusieurs valves isolées, max. long. $14 \mathrm{~mm}$. Elles ressemblent en formes et en sculpture à la fig. 115 de ReEve (Conch. Tcon. 2). Soit que l'ex. figuré semble être un peu plus court et plus haut, la sculpture est si caractéristique qu'on ne pourrait douter de l'identité. Les ex. présents montrent comme dans la figure citée, à la moitié antérieure des valves, des costules radiantes serrées, d'épaisses et de larges alternativement, qui s'élargissent à la moitié postérieure en se bifurquant. Aux côtés des cordons épais l'épiderme forme des squámules pointues, tout à fait comme l'écrit ReEve en ces mots: 》lines of fine dark hair rising from between the crenulations of about every third rib». - LAMY (1907) discute la synonymie et la distribution de cette espèce.

Pectunculus radians Lamarck. Majunga, la plage, $2 \mathrm{v}$. g., max. long. $27 \mathrm{~mm}$. De cette espèce il se trouvent deux ex. dans les coll. du R. M., qui font voir le caractère spécifique: des côtes à fines stries longitudinales. Cette propriété se trouve aussi chez la forme présente qui a, de plus, le contour oblique de la forme d'Australie, l'extrémité postérieure étant un peu allongée. Elle diffère par ses côtes moins serrées; chez la forme d'Australie il y a 33 côtes en tout, tandis qu'il n'y en a que 28 chez la forme de Madagascar. Une chose caractéristique pour l'espèce est la courbure presque anguleuse que comprennent les deux parties du plat denticulair s'étendant en ligne droite sur les deux côtés des sommets. Le ligament est très court $(2 / 3$ du plat denticulaire) et les bords supérieurs s'inclinent en bas immédiatement aux extrémités du ligament.

$P$. pertusus Reeve. Majunga, dragage, une trés petite valve, long. $4 \mathrm{~mm}$, d'une sculpture consistant en côtes rayonnantes flanquées de séries de points impressés; sommets médians. 
Lucina (Phacoides) semperiana Isser. Majunga, dragage, 1 v.g. + 1 v.dr., max. long. $5 \mathrm{~mm}$. Quoique usés, ces exemplaires sont tout à fait déterminables et se rapportent à l'espèce typique, présente aux collections de R. M. (originale de M'ANDREw).

Diplodonta subcostata n. sp. Pl. 1, fig. 2, 3. Majunga, dragage, 1 v. dr., long. 8,6, hauteur $7 \mathrm{~mm}$. Coquille presque circulaire, à crochets médians, largement arrondie en arrière; un peu plus allongée en avant; bord ventral uniformement convex et formant au bord postérieur un coin indistinct. Couleur grise-blanche, faiblement lustrée. Sculpture consistant en stries concentriques, irrégulières et serrées, coupées de stries rayonnantes peu visibles, apparaissant ça et là et formant une granulation très mince sur toute la coquille. Une carène peu marquée s'étend des crochets au coin postéro-inférieur et au-dessus d'elle quelques-unes plus minces; aussi en avant des crochets se trouvent des traces d'une semblable carène indistincte. Charnière à deux dents cardinales et une latérale antérieur; la fosse ligamentaire courte, deux fois plus longue que la dent postérieure.

Par ses crochets médians et petits, pas renflés, cette espèce diffère d'un côté de la $D$. ethima Melvill, et de l'autre de la $D$. raveyensis Sturany, de laquelle elle se rapproche, cependant, par la longueur relative du ligament.

Scintilla symmelrica n. sp. Pl. 1, fig. 4. Majunga, dragage, 1 v.dr., long. 10, haut. 6,4, larg. $2 \mathrm{~mm}$. Coquille hyaline, assez renflée, mais pas ventrue, d'une forme ovale-étendue; bords antérieurs et postérieurs arrondis, de plus vers le bas, bord inférieur modérément convexe, seulement au milieu faiblement fléchi en dehors. Crochets médians. Surface fortement brillante, irrégulièrement rugueuse d'une manière concentrique et striée de très indistinctes lignes rayonnantes; la sculpture donne une apparence presque comme martelée. Charnière à deux dents cardinales rapprochées, et une fosse ligamentaire étendue.

Tellina madagascariensis GMeLıN. Majunga, dragage, 10 valves de jeunes ex., max. long. $24,5 \mathrm{~mm}$. 'Bien que petits, ces ex. font apparaître plus ou moins distinctement les côtes obliques postérieures caractéristiques à l'espèce, qui sont 
visibles à la fig. 18 de Reeve (Conch. Icon. 17, Tellina). Quelques ex. de la $T$. lux appartenant aux collections de M'AnDREw de Suez sont assez semblables mais tout à fait lisses, pas concentriquement striées, et d'une couleur plus claire.

T. semitorta Sowerby. Majunga, dragage, 1 v. g. +1 v. dr., max. long. 13,5 mm. Pour détails de cette espèce voir p. 27.

Eastonia africana BARTSCH 1915. Majunga, dragage, 2 v. g. +1 v. dr., max. long. $10,5 \mathrm{~mm}$. La forme des valves gauches est semblable à celle des fig. 5 et 6 , pl. 43 , chez Bartsch, mais la valve droite est plus allongée et les costules sont plus espacées, rappellant la Mactra (Standella) solandri chez Reeve (Conch. Icon. 8, fig. 113). Ces deux formes sont probablement identiques; l'Eastonia africana pourrait être une forme juvenile de l'E. nicobarica GMeLIN (= Mactra cegyptiaca Reeve, Conch. Icon. 8, sp. 112).

Semele radiata RüPpell. Majunga, dragage, 2 v. g., max. long. $21 \mathrm{~mm}$. La forme et la sculpture sont complètement concordantes avec celles de la fig. 12 (Conch. Icon. 8, Amphidesma) de ReEve, mais les valves sont décolorées: néanmoins, des traces de rayons rougeâtres sont encore visibles sur la coquille la plus grande, et à cause de cela, je réfère les ex. à cette espèce de RüPPELL.

Theora lata Hinds. Majunga, dragage, plusieurs valves, $\max$. long. 14,5 mm. Les ex. correspondent à la figure des H. \& A. Adams (1858, Genera of Recent Mollusca, Pl. XCVII, fig. 5) et à ceux de LYNGe 1909 (Tab. IV, fig. 10-11); le dernier auteur réunit avec cette espèce la $T h$. fragilis $\mathbf{A}$. Adams. Selon Lamy $1913^{1}$ le genre comprend 8 espèces outre la présente.

Donax elegans n. sp. Pl. 1, fig. 5-8. Majunga, la plage, plusieurs valves, max. long. 20, haut. 15, larg. (crass.) d't.ne valve $4,5 \mathrm{~mm}$. Coquille cunéiforme, la partie antérieure allongée et également arrondie en avant, la partie postérieure très courte, obliquement tronquée; bord ventral modérément

${ }^{1}$ Revision des Scrobiculariidae vivants. Journ. de Conch. 
convexe. Surface de la partie antérieure lisse, très Juisante, celle de la partie postérieure divisée en deux, la première partie sculptée de costules rayonnantes et s'accroissant vers l'inférieur; la seconde partie située près du bord postérieur, réticulée de fines costules rayonnantes découpées par des lamelles concentriques relevées, formant la continuation des stries d'accroissement. Couleur variant de zones concentriques blanchâtres et bleues sous un épiderme brun-jaunâtre et quelques larges rayons de la même couleur s'étendant des crochets vers le bas; l'intérieur plus ou moins coloré de violet avec un rayon blanc distinct tout en avant de l'impression musculaire postérieure et deux taches jaunes allongées en arrière de l'impression antérieure. Bord inférieur distinctement crénelé.

La forme de cette espèce l'approche d'un côté du $D$. $t i$ caonicus (Reeve, Conch. Icon. 8, fig. 13 , et Römer, Martini \& Chemnitz, Conch. Cab. 10: 3, pl. 18, figs. 1-4) et de l'autre du D. incertus Bertin 1881, ${ }^{1}$ mais la sculpture la sépare de tous les deux, comme la coquille est, d'un côté, dentelée au bord ventral, tandis que le D. ticaonicus y est lisse (selon un ex. à R. M.); outre cela, le $D$. incertus a seulement des rides longitudinales granuleuses, mais pas de lamelles concentriques. Comme elle ne s'accorde à aucune forme connue par moi, j'ai décidé de la décrire comme nouvelle espèce.

Mactra lurida Philippi. Majunga, dragage, 1 valve gauche, long. $21 \mathrm{~mm}$. Cet exemplaire concorde par sa forme fortement renflée et ses obscurs rayons brunâtres avec la fig. 96 de Reeve (Conch. Icon. 8), mais la moitié supérieure de la coquille est marbrée et les crochets sont violets comme chez $M$. achatina; l'intérieur est en outre également brunâtre, pas violet comme chez la $M$. lurida typique. Il est évident que l'ex. présent est un grand individu de cette espèce en le comparant avec des exemplaires de Tamatave.

Corbula acutangula Issel. Majunga, dragage, 2 valves gauches, max. long. $18 \mathrm{~mm}$. Le plus grand d'eux correspond en détail à fig. 1, Tav. III, chez IsseL (1869). ${ }^{2}$ Les caractères

${ }^{1}$ Révision des Donacidées du Mus. d'Hist. nat. Nouvelles Arch. du Mus. d'Hist. nat. Paris. Sér. II, Tome IV.

"Malacologia del Mar Rosso. Pisa. 
spécifiques sont les forts plis, en nombre de 17-18, qui sont sculptés et par 3 sillons de chaque côté; par ce fait cette espèce se sépare de la $C$. crassa, qui a plusieurs côtes et entre eux une réticulation très fine de stries concentriques et radiantes. C. scaphoides est une autre espèce semblable, mais elle a les interstices longitudinalement striées. L'espèce devant nous est probablement identique à la $C$. taheitensis Lamarck, que M. E. A. Smith $(1891)^{1}$ a signalée d'Aden. Isser l'a récoltée au milieu de spécimens fossiles de la mer Rouge.

Chione siamensis LYNGE 1909. Majunga, dragage, 1 valve droite, long. 5,2 mm. Bien qu'elle n'est pas adulte, la valve présente offre tous les détails qui caractérisent l'espèce à en juger par la figure et la description de Lyngs (1909, Tab. V, fig. $6-7$ ). La sculpture caractéristique rayonnante qui est formée par des costules plus serrées au milieu de la coquille, plus espacées et épaisses en arrière, et qui sont absentes à l'aire lisse, est si distincte dans la forme présente que l'identité est indiscutable. Comme l'espèce est décrite de Siam, où elle fut draguée à 30 brasses de profondeur, elle a certainement une distribution dans la mer des Indes plus étendue que celle connue jusqu'ici.

Gaffrarium pectinatum Lınné. Majunga, la plage, 6 valves, max. long. $30 \mathrm{~mm}$.

Cardium rugosum Lamarck. Majunga, la plage, 1 valve gauche, haut. $20 \mathrm{~mm}$. Bien que petit, l'exemplaire présent appartient avec certitude à cette espèce à en juger par la forme qui est étendue en direction dorso-ventrale, et par la sculpture, Les 6 côtes postérieures sont un peu plus fines que les précédentes et portent des épines squamiformes plus espacées, caractères qui sont ceux du type (ex. à R. M.).

C. dupuchense Revve. Majunga, la plage, 1 valve gauche, long. $29 \mathrm{~mm}$. Par sa forme plus circulaire cet exemplaire diffère de $C$. rugosum et s'accorde avec un exemplaire de $C$. dupuchense de la Nouvelle Calédonie à R. M. Comme à cette coquille les 7 costules postérieures portent des épines squami-

${ }^{1}$ Proc. Zool. Soc. London. 
formes espacées. La valve indique aussi des traces de la coloration caractéristique qui consiste en taches éparses rondes et noires sur les côtes de la partie postérieure, quoique les taches de l'exemplaire en question se trouvent, au contraire, sur les côtes médianes. La forme est montrée par la fig. 67, pl. XIV, de Reeve (Conch. Icon. 2). Jousseaume (1888) ${ }^{1}$ constitue pour cette forme une espèce distincte, $C$. peregrinum, qui serait différente du $C$. dupuchense par »sa forme oblique et un peu aplatie et la présence des taches noires de la surface», des caractères auquels je ne puis attribuer aucune signification spécifique, d'autant plus que la sculpture de l'exemplaire de la Nouvelle Calédonie et celle de la figure de Reeve sont conformes.

C. coronatum Spengler. Majunga, la plage, 1 valve droite, long. $29 \mathrm{~mm}$, d'après la comparaison avec d'autres exemplaires de cette espèce à $\mathbf{R}$. M.

Hemicardium retuisum Linné. Majunga, la plage, 1 valve, droite, long. $20 \mathrm{~mm}$, usée.

Tridacna elongata Lamarck. Mahakamby, aug. 1912, 1 ex., long. $180 \mathrm{~mm}$.

Chama spinosa Broderip. Majunga, dragage, 1 valve droite, long. $12 \mathrm{~mm}$. Cette petite valve ressemble un peu à la Ch. aspersa ReEve par sa sculpture consistant en des squamules très serrées, mais la comparaison à des échantillons de Tamatave prouve l'appartenance à cette espèce.

Cultellus grayanus Dunker. Majunga, dragage, 6 valves, max. long. $22 \mathrm{~mm}$. Cette forme ne correspond pas tout à fait à la fig. 17 de ReEve (Conch. Icon. 19, Cultellus), parceque la partie antérieure en est un peu plus allongée que dans cette dernière bien que largement arrondie. Mais la faible convexité du bord dorsal et la coloration ainsi que la forte côte intérieure offrent des caractères qui font probable l'identité. Elle est, cependant, un peu incertaine, n'ayant pas eu à ma disposition des exemplaires typiques pour faire une comparaison. Les valves sont très fragiles.

1 D scription des Moll. rec. dans la Mer Rouge et le Golfe d'Aden. Mém. Soc. Zuol. France 1. 
Petricola ventricosa Krauss. Majunga, dragage, 2 valves droites, max. long. $13 \mathrm{~mm}$.

Dentalium aprinum Linné. Majunga, la plage, 2 coquilles décolorées, max. long. $52 \mathrm{~mm}$, diam. 5,5 mm. La sculpture typique, composée de 12 côtes sur la petite et 10 sur l'autre, les plus grandes au côté concave, et des intermédiaires très indistinctes.

Helcioniscus rota Gmelin. Majunga (2/4), 1 ex., long. 5 mm. La coloration est formée de 9 larges rayons noirs. La partie apicale est sculptée de costelets rayonnants et dessinée de courtes raies rouges longitudinales. - Mahakamby, 1 ex., long. $41 \mathrm{~mm}$.

Glyphis rüppelli Sowerby. Majunga, dragage, 2 coquilles, max. long. $11 \mathrm{~mm}$.

Clanculus puniceus PhiLippi. Mahakamby, 1 ex., haut. $13 \mathrm{~mm}\left({ }^{26} / 8\right)$.

Turbo coronatus GMelin. Mahakamby, 10 ex., max. diam. $35 \mathrm{~mm}$, haut. $30 \mathrm{~mm}$. La forme est semblable à la fig. 60 , pl. 50, de Pilsbry (Man. of Conch., Vol. 10), mais la sculpture ressemble plus à celle de la fig. 18, pl. 46 ; il y a seulement une série de tubercules subsuturaux qui sont pressés tout au tour précédent; pour le reste la sculpture générale noduleuse est prévalente.

Nerita undata Linné. Majunga, la plage. 1 ex., haut. $22 \mathrm{~mm}$. - Mahakamby, 1 ex., h. $28 \mathrm{~mm}$. - Amborovy, 5 ex., $\max .32 \mathrm{~mm}\left({ }^{28} / 8\right)$.

N. albicilla Linné. Majunga, la plage, 2 ex., long. 30 $\mathrm{mm}$; dragage, 1 ex., long. $20 \mathrm{~mm}$; les rocs de la côte, 3 ex. $(7 / 6$ 1912), max. long. $25 \mathrm{~mm}$.

N. plicata Linné. Amborovy (17/4), 1 ex., haut. $23 \mathrm{~mm}$.

Natica mamilla Linné. Majunga, 3 ex., max. long. $34 \mathrm{~mm}$.

N. melanostoma Gmelin. Majunga, la plage, 1 ex., long. $40 \mathrm{~mm}$. 
N. ala papilionis Chemnitz. Majunga, la plage, 1 ex., haut. $18 \mathrm{~mm}$.

Torinia variegata Gmelin, var. planulata Hanley. Majunga $(\% / 4), 1$ ex., diam. $8 \mathrm{~mm}$. von MARTEns (1880) constate l'espèce (le type) à Madagascar. L'exemplaire présent concorde bien avec la fig. de Tryon (Man. of Conch., vol. 9, 1887, pl. 5, fig. 77).

Hipponyx ticaonicus Sowerby. Majunga, la plage (12/9), 1 ex., long. 17,5 mm. Ouverture orbiculaire, sommet étendu fort en arrière, placé vers la droite, sculpture consistant en fines lignes élevées radiantes, découpées de stries d'accroissement, cuticule villeuse, brunâtre. L'exemplaire correspond bien aux figs. 23, 24, pl. 41, chez Tryon, 1886 (Man. of Conch., vol. 8). L'espèce était connue auparavant aux Philippines, mais, selon Tryon, est peut-être identique à $H$. subrufus des mers des Indes occidentales.

Cerithium columna Sowerby. Majunga ( $\left.{ }^{2} / 4\right), 2$ ex., long. $23 \mathrm{~mm}$.

C. morus Lamarck. Majunga $(2 / 4), 5$ ex., max. long. $23 \mathrm{~mm}$.

Triphora hindsi Deshayes. Majunga, dragage, 1 ex., long. $7 \mathrm{~mm}$. Ses 3 séries de petits tubercules, dont la médiane est la plus étroite, causent une ressemblance avec la fig. 19 de Deshayes (1863, pl. 38). Sur le dernier tour se trouvent quatre séries de tubercules au lieu de trois, comme le dit Deshayes. L'espèce était jusqu'ici connue seulement à l'île de la Réunion.

Potamides palustris Linné. Majunga, les rochers, 2 ex., haut. $96 \mathrm{~mm}$, connu auparavant à Madagascar selon v. MaRTENS (1880).

Siliquaria trochlearis Мöвcн. Majunga, dragage, 1 petite coquille (sommet), diam. 7,5 mm; les costules serrées prouvent qu'elle appartient à cette espèce.

Littorina scabra Linné. Antanamasadza, Majunga (11/4), plusieurs ex., très variés en couleur, max. haut. $20 \mathrm{~mm}$, parmi des massifs de mangliers. 
Strombus auris diance Linné. Majunga, la plage, 1 ex., haut. $32 \mathrm{~mm}$.

S. labiosus Gray. Majunga, 1 ex., long. $35 \mathrm{~mm}$.

Pyramidella (Elusa) subglabra n. sp. Pl. 1, fig. 9. Majunga, dragage, 1 ex. Coquille étroitement turriculée, sculptée de costules longitudinales très serrées, à peu près visibles, surface pour le reste lisse et luisante sans aucune sculpture. Une bande spirale brunâtre immédiatement au-dessus de la suture et au milieu du dernier tour, et une zone grisâtre au-dessous de la suture. Péristome continu. Ouverture étroitement rhomboidale, un peu prolongée à la base de la columelle et formant ici une trace de siphon; bord columellaire très étendu, limitant un ombilic sillonné et portant un pli presque médian. Dimensions: long. 5,6, larg. 1,2 mm, tours 11.

Cette espèce ressemble à $P$. gracilis A. Adams de la mer Rouge (ex. à R. M. comparés), mais elle en diffère par sa taille plus petite, ses faibles costules et la forme de l'ouverture et celle de la columelle retournée.

Vasum turbinellum LinNé. Mahakamby (aug. 1912), 10 ex., max. haut. $72 \mathrm{~mm}$.

Ranella granifera Lamarck. Majunga $(2 / 4), 4$ ex., max. haut. $55 \mathrm{~mm}$.

Nassa coronata Bruguik̀re. Majunga, 1 ex., haut. $15 \mathrm{~mm}$.

N. sinusigera A. Adams. Majunga, dragage, 2 ex., max. haut. $9,5 \mathrm{~mm}$, caractérisés par le sinus à la base du rebord.

$N$. arcularia Lamarck. Majunga, 3 ex., max. long. $21 \mathrm{~mm}$.

Columbella flava Bruguière. Majunga ( $7 / 6), 1$ ex., haut. $15 \mathrm{~mm}$; couleur principale brune, de grandes taches claires à la suture et au bas du milieu des tours, pour le reste faiblement marbrée par la teinte claire.

C. alabastrum v. Martens. Majunga, dragage, 4 ex. Ils sont caractérisés par une série de taches blanches (ou par une ligne blanche interrompue) immédiatement au-dessus de la suture (parfois absente) et de quelques taches brunes plus 
ODHNER, LA FAUNE MALACOLOGIQUE DE MADAgascar. 17

grandes inférieures à elle qui peuvent être absentes aussi. Couleur principale claire jaune-brunâtre. Par ces caractères les ex. correspondent le mieux à la fig. 13, pl. XX, de v. Martens 1880. Le $C$. alabastrum de Reeve me semble être une espèce différente.

Cyproea onyx Linné. Majunga, 1 ex., long. $40 \mathrm{~mm}$. Couleur isabelle avec une large bande transversale un peu plus foncée; les côtés, les extrémités et la base chocolat foncées.

C. lamarcki Gray. Majunga, 2 ex., max. long. $38 \mathrm{~mm}$.

C. caput serpentis Linné. Majunga, 4 ex., max. long. $36 \mathrm{~mm}$.

C. erosa Linné. Majunga, la plage, 3 ex., max. long. $32 \mathrm{~mm}$. - Amborovy (28/8) 2 ex., max. long. $28 \mathrm{~mm}$.

C. caurica Linné. Majunga, la plage, l ex., long. 41 mm. - Amborovy, 3 ex., max. long. $34 \mathrm{~mm}$. - Mahakamby $(26 / 8), 1$ ex., long. $37 \mathrm{~mm}$.

C. annulus Linné. Mahakamby (26/8), plusieurs ex., max. long. $23 \mathrm{~mm}$; les jeunes individus ont deux bandes transversales d'une couleur isabelle.

Peristernia chlorostoma Sowerby. Majunga, 1 ex., baut. $24 \mathrm{~mm}$.

Pyrula ficus Linné. Majunga, la plage, 2 ex., max. long. $47 \mathrm{~mm}$.

Fasciolaria trapezium Linné. Mahakamby, 4 ex., max. long. $155 \mathrm{~mm}$, avec des Balanidés.

Melongena paradisiaca Reeve. Majunga, sur les rochers, 1 ex., haut. $53 \mathrm{~mm}(7 / 6$ 1912). Unicolore de jaune brunâtre sans bandes et sans tubercules sur les tours, dans l'ouverture une bande longitudinale brune près du bord externe interrompue par les sillons spiraux intérieurs.

Mitra cylindracea Revve. Majunga, sur les rochers calcaires ( $7 / 6$ 1912), 1 ex., long. $43 \mathrm{~mm}$. La couleur principale de la cuticule olive aux raies courtes, jaunes claires, ou aux 
taches irrégulières, étroites, au-dessous de la suture, situées sur une bande plus foncée; l'indication d'une bande obscure à la périphérie du dernier tour; les stries spirales fines vers le sommet, en bas plus fortement sculptées; 4 plis de columelle, dont l'inférieur est, cependant, très faible; la couleur est blanche brunâtre dans l'ouverture qui est un peu contractée vers la base. Tous ces caractères rapprochent l'ex. présent de la $M$. cylindracea ReEve, de laquelle il diffère, cependant, par sa largeur plus grande relativement à la longueur. Si l'on considère avec Tryon (1882) M. cylindracea REEVE comme synonyme de la $M$. variabilis $R$ Eeve, quoique elles diffèrent assez fortement en couleur et en forme, il faut aussi rapporter l'exemplaire en question à $M$. cylindracea, malgré la différence qu'il présente avec la forme typique (Conch. Icon. 2, fig. 97).

Murex torrefactus Sowerby. Majunga (2/4 1912), 4 ex., max. long. $53 \mathrm{~mm}$. Ils ressemblent le mieux à $M$. rubiginosus Reeve (Conch. Icon. 3, fig. 32), qui est considéré par Tryon Man. of Conch. 1880) comme synonyme à $M$. torrefactus. $48 \mathrm{~mm}$.

M. ternispina Lamarck. Majunga, la plage, 1 ex., long.

$M$. ramosus Linné. Mahakamby, 4 ex., avec des animaux, max. long. $150 \mathrm{~mm}$, couverts par des Ostrées et par des Balanidés.

Purpura bufo LAmarck. Majunga, sur les rocbers calcaires, plusieurs ex., max. long. $43 \mathrm{~mm}$. La variation est très marquée, puisque chez les ex. de grande taille la coquille est plus épaisse, la columelle orange et le bord extérieur de même couleur; chez des ex. plus petits le bord extérieur est brun à costules blanches, la columelle est plus claire et la callosité à l'angle supérieur de l'ouverture est plus faible; la spire est aussi plus haute relativement à l'ouverture.

Ricinula undata Chemnitz. Majunga ( $2 / 4$ 1912), 2 coquilles, max. haut. $18 \mathrm{~mm}$ (en partie var. albovaria KüsTER). Fénérive, les rochers, 1 ex., haut. $10 \mathrm{~mm}$.

R. tuberculata Blainville. Majunga ( $2 / 41912)$, plusieurs ex., max. haut. $23 \mathrm{~mm}$. 
R. ochrostoma Blainville, var. heptagonalis Reeve. Majunga $(2 / 4$ 1912), ca. $14 \mathrm{ex}$., max. haut. $24 \mathrm{~mm}$, concordant avec fig. 34 chez Reeve (Conch. Icon. 3, R. ocellata), forme laquelle Tryon 1880 range avec la var. heptagonalis Revve.

Oliva inflata Lamarck. Majunga, la plage, 9 ex., max. long. $35 \mathrm{~mm}$ à un nombre de tours de $5^{1 / 4}$.

O. tigrina Lamarck. Majunga, la plage, 4 ex., max. long. $48 \mathrm{~mm}$ à un nombre de tours de $5^{1 / 2}$.

O. mustellina Lamarck. Majunga, la plage, 3 ex., max. long. $25 \mathrm{~mm}$, ressemblant aux figures de pl. 22 chez Tryon 1883 (= O. caroliniana Duclos chez v. Martens 1880).

Drillia griffithii Reeve. Majunga (2/4), 1 ex.. haut. 25 $\mathrm{mm}$. Il ressemble à la fig. de la Pleurotoma griffithii chez Reeve (Conch. Icon., 1, fig. 57), qui est référée par Tryon (1884) à la $P$. crenularis LAMARck au mépris de la forme et de la couleur différente entre les deux (voir ReEve, Conch. Icon. 1, fig. j4).

Conus striatus Linné. Majunga, la plage, l ex. décoloré, long. $83 \mathrm{~mm}$.

C. hebraus Linné. Mahakamby, 1 ex., long. $30 \mathrm{~mm}$.

C. textile Linné. Majunga, la plage, 2 ex., max. haut. $55 \mathrm{~mm}$; les rochers calcaires $(7 / 6), 1$ ex., haut. $46 \mathrm{~mm}$.

C. gubernator Hwass. Amborovy, 1 ex., haut. $61 \mathrm{~mm}$.

Cavolinia longirostris Lesueur. Majunga, dragage, 1 ex., long. $4 \mathrm{~mm}$.

Atys naucum Linné. Majunga, la plage, 1 ex., haut. $32 \mathrm{~mm}$.

Aplysia tigrinella Gray. Amborovy (28/8 1912), 1 ex., long. 21, coquille $13,5 \mathrm{~mm}$. L'exemplaire présent correspond essentiellement aux figs. 5, 6, pl. 16 chez Pilsbry (Man. of Conch. vol. XVI, 1895-6), reproduites de Quoy \& Gaimard. Le caractère spécifique est le revêtement extérieur des parapodes par des appendices papilliformes; ils sont uniques 
dans le genre Aplysia et au contraire significatifs pour le genre Notarchus. L'ex. présent porte des papilles aussi sur les côtés et l'extrémité du pied. Par dessus, la coquille a une forme différente, son contour étant sinueux dans la moitié postérieure du côté droit, non pas ovale, comme montre la figure de Prlsbry. La couleur semble avoir été également verte claire. Les yeux sont placés extérieurement aux tentacules postérieurs. Le manteau n'est pas non plus ouvert si loin en arrière. Cependant, comme les papilles, qui sont absentes à toutes les autres espèces de ce genre, et la couleur sont communes à l'original de QuoY \& GaImaRd, l'individu présent, bien qu'un peu aberrant, doit être rapporté à cette même espèce. Le type a été trouvé à Mauritius.

Siphonaria madagascariensis n. sp. Pl. 1, fig. 10-12. Majunga, les rochers $(19 / 4$ 1912), plusieurs ex. Coquille déprimée, ovale, plus ou moins allongée; sommet post-central, placé à gauche. Sculpture composée de côtes radiantes épaisses, alternant avec d'autres plus étroites; les plus fortes montant jusqu'à 20 en nombre, moins nombreux sur le côté droit que sur le côté gauche; celles-là sont les plus fortes, et les médianes renferment une aire triangulaire, limitée en avant par une côte double. Toutes les.côtes irrégulièrement tubuleuses, découpées par des stries d'accroissement atteignant presque la forme de lamelles. L'intérieur de la coquille lisse, sillonné vers les bords. Couleur extérieurement grise tirant au brunâtre, intérieurement brune claire, luisante, avec un anneau musculaire un peu plus foncé et des taches éparses d'un brun foncé, correspondant aux interstices des côtes, ça et là vers les bords des lignes. brunes foncées. - (Quelques taches blanches sont répandues à l'intérieur, causées par de petites vesicules situées intérieurement à la couche interne, formées par des parasites?). Dimensions: Long. 16,5, larg. 11,3, haut. $4,5 \mathrm{~mm}$, ex. figuré; un autre échantillon: long. 15,6, larg. 12,6 , haut. $4,3 \mathrm{~mm}$. La forme, en conséquence, est assez variée.

Asteronotus mabilla Bergh. Amborovy ( ${ }^{28} / \mathrm{s} 1912$ ), 1 ex., long. $1 \mathrm{dm}$. Selon Bergh $1880^{1}$ l'espèce habite les Seychelles et l'île de Samoa.

${ }^{1}$ Malak. Unters. Suppl. H. 1. Reisen im Archipel der Philippinen. II: 2, Wiesbaden. 
Discodoris concinna AL.DER \& HANCOCK (= concinniformis Bergh). Mahakamby $\left({ }^{26} / 8\right.$ 1912), 1 ex., long. $56 \mathrm{~mm}$. Cet exemplaire semble être un intermédiaire quant à la couleur de $D$. concinniformis et de la variété de cette espèce qu'a décrite BERGH (1880). ${ }^{1}$ La surface dorsale est grisâtre mêlée de taches brunes d'une forme irrégulière, arrondies ou allongées, souvent d'une couleur plus foncée accentuée à la périphérie; elles composent une sorte de deux bandes latérales sur la face dorsale; l'inférieur du manteau montre une zone brune-foncée près du côté du pied et un limbe grisâtre; la sole du pied est tachetée de brun, avec un bord à petites taches grisâtres; la face supérieure des côtés du pied est aussi tachetée de brun.

Acanthopleura spiniger SowerBy. Mahakamby (26/7 1912), 3 ex., max. long. ca. $60 \mathrm{~mm}$.

Ischnochiton rufopunctatus n. sp. Pl. 3, fjg. 40, 41. Animal allongé elliptique à côtés presque parallèles, le dos faiblement caréné. Valves à aires latérales distinctes (sauf la première), la seconde un peu plus large que les autres, prominente au milieu et amincie aux parties latérales, les autres valves à longueur uniforme sur toute leur largeur. Valve antérieure à 10 fissures; la postérieure à 8 , déprimée, à sommet peu élevé, subcentral. Lames d'insertions des valves intermédiaires à bord unifissuré. Sculpture: aires médianes réticulées, finement au sommet, plus rudement en dehors; aires latérales densement granuleuses. Les creux des mailles des aires médianes et les interstices entre les granules latéraux d'une couleur rouge-brune. L'intérieur des valves blanc. Zone mince à couleur rouge-brune, la surface supérieure imbriquée d'écailles finement striées (long. 0,3, larg. 0,2 mm, environ; plus minces et étroites vers le bord; fig. 1); la surface inférieure revêtue d'écailles en forme de petits bâtons tronqués (long. 0,1, larg. 0,03 mm environ; fig. 2). Pas de frange marginale. Branchies occupant toute la longueur de la rainure parapodiale. Localité: Majunga ( ${ }^{14} / 4$ 1912), 1 ex., long. 7 , larg. $3,5 \mathrm{~mm}$.

${ }^{1}$ Malak. Unters. H. XVI, Nudibranchien vom Meere der Insel Mauritius. Sempers Reisen, II: 2 . 
Acanthochites aberrans n. sp. Pl. 3, fig. 42, 43. Corps oval. Valve antérieure étroitement semi-lunaire, à 5 fissures dans le bord, correspondant à 5 rayons d'incisures sur la face supérieure de l'articulamentum. Valves intermédiaires unifissurées, à sommets proéminents postérieurement; valve postérieure courbée, deux fois plus courte que l'antérieure, profondement sinueuse en avant et produite en forme d'ailes aux côtés, à 5 incisures marginales; mucro indistinct, submédian. Tegmentum divisé en une aire dorsale et deux aires latéro-pleurales. Couleur brunâtre tirant sur le rouge au côtés, blanchâtre sur l'aire dorsale (au moins vers le sommet), qui est séparée des latérales par des lignes ou des séries de taches olivâtres. Sculpture consistant sur toute la valve en granules ovales, plus serrés et plus petits au dos qu'aux parties latérales et arrangées en séries indistinctes. L'inté-

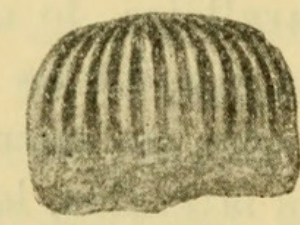

Fig. 1.

Ischnochiton rufopunctatus $\mathrm{n}$. $\mathrm{sp}$.

Ecaille de la surface supérieure de la zone.

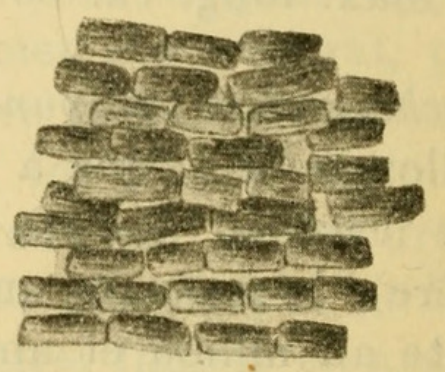

Fig. 2.

Ischnochiton rufopunctatus $\mathrm{n}$. $\mathrm{sp}$. Revêtement d'écailles de la surface inférieure de la zone.

rieur des valves blanc, le brun paraissant à travers. - La zone de la demi-largeur du pied marbrée de brun et olivâtre, à pores de fascicules entre les valves (4 devant la première valve), revêtue densement aux deux côtés de spicules aciculaires et lisses. Branchies étendues à la demi-longueur du pied. Long. 4, larg. 2,5 mm. Localité: Majunga $(14 / 41912)$.

La sculpture de cette espèce (pl. 3, fig. 43) rappelle celle d'un Craspedochiton, mais la forme et l'étendue de la zone ainsi que son revêtement de spicules (non pas d'écailles) montrent qu'elle appartient à l'Acanthochites. Les formes de ce genre, dont la ressemblance est la plus grande, semblent être $A$. carpenteri Pilsbry et $A$. involutus Carpenter de la côte orientale de l'Afrique, qui ont une sculpture et une valve postérieure rappelant celles de l'espèce présente(PILSBRY, Man. of Conch. Vol. 15, 1893, pl. 1, figs. 14-22, 27-35), 
mais qui, de l'autre côté, diffèrent considérablement quant à l'étendue relative du pied et du notum.

Oncidium verruculatum Cuvier. Catsèpe (12/5 1912), dans des lagunes à mer basse, 2 ex., max. long. $33 \mathrm{~mm}$. Tous les deux exemplaires font apparaître très distinctement les caractères mentionnés par SEMPER, $1880 ;^{1}$ les papilles branchiales apparaissant vers l'extrémité postérieure et les yeux dorsaux plus nombreux vers le bord antérieur, pendant que ceux-ci disparaissent à la partie postérieure, où se trouvent les papilles. La couleur est claire fauve avec des taches et des nuages noirâtres.

\section{Mollusques marins de la côte de l'est.}

La localité de ces collections est Tamatave, où elles ont été faites pour la plupart sur le récif de coraux ou par dragage entre ce dernier et la côte.

Pecten pyxidatus Borw. Tamatave, dragage ( $2 / 3$ 1912), 2 valves droites et 3 gauches, max. haut. $18 \mathrm{~mm}$. Concordant avec la fig. $96 a$ de Reeve (Conch. Icon. 8). Pas auparavant mentionné de Madagascar. Distribution: Philippines-Golfe Persique (Lynge 1909).

Meleagrina (Pteria) margaritifera Linné. Tamatave, sur le récif de coraux ( $28 / 1$ 1912), 1 ex., haut. $16 \mathrm{~mm}$.

Nucula rugosa n. sp. Pl. 2, fig. 15-18. Coquille allongée ovale avec des crochets assez saillants, placés fortement en arrière. Bord supérieur faiblement convexe, formant sur le front un angle arrondi avec le bord inférieur fortement courbé; celui-ci passe en arrière, par un coin prononcé, dans le bord postérieur qui est droit à la base, convexe au-dessus. Sculpture composée de fortes côtes concentriques irrégulièrement successives, qui sont érigées en forme de lamelles vers les crochets, plus épaisses et s'inclinant vers l'inférieur; l'un ou l'autre est courte et se termine plus vite. Les côtes sont ca. 35 en nombre. A leur côté inférieur elles sont pourvues de stries

${ }^{1}$ Landmollusken. H. V. Reisen im Archipel der Philippinen. II: 3. Wiesbaden. 
radiantes indistinctes et assez espacées. Les sommets de la coquille sont lisses. Lunule et corselet bien limités par des dos et des sillons plus ou moins marqués, et sculptés de costules transversales, qui forment la suite des côtes concentriques, mais qui sont fléchies par les sillons. Couleur verte brunâtre. Dents postérieures 9, antérieures 22-24. L'intérieur fortement nacré, bord inférieur crénelé, Ligament dirigé obliquement en avant. Dimensions (max.): long. 13,5, haut. (perpendiculairement à la longueur) 10,5 , crassitude $7,9 \mathrm{~mm}$. Localité: Tamatave ( ${ }^{2} / 3$ 1912), dragage, plusieurs ex.

L'espèce ressemble à la $N$. pulchra REeve (Conch. Icon. 18, fig. 38) de l'Afrique du sud, qui a, cependant, le corselet lisse, et à la $N$. sculpta Sowerby $\left(1908,{ }^{1}\right.$ fig. 11, pl. VI), aussi de l'Afrique du sud; mais la taille de celle-ci est plus haute, tandis que les dents sont peu nombreuses $(7+17)$. Ni v. Martens ni Lamy n'a cité aucune Nucula de Madagascar.

Yoldia divaricata n. sp. Pl. 1, fig. 13, 14. Tamatave, dragage $(2 / 3)$, 1 valve gauche, long. 12,7 ; haut. 7 ; larg. $2,3 \mathrm{~mm}$. Coquille d'une couleur blanche - jaune; les sommets un peu en avant du milieu; partie antérieure allongée et arrondie; bord postéro-supérieur entièrement droit formant un angle arrondi avec le bord postérieur, qui est faiblement convexe; bord inférieur fortement courbé. Sculpture consistant en fines stries serrées, dirigées obliquement au postéro-inférieur, laissant lisse une aire étroite près du bord postéro-supérieur. Tout près $d u$ bord antérieur les stries se fléchissent en haut, formant un sinus, et puis continuent, la direction un peu changée, vers le bord. Dents ca. 17 devant ainsi que derrière la fosse ligamentaire triangulaire.

Cette espèce ressemble en forme d'un côté à $Y$. serotina A. Adams et de l'autre à $Y$. tropica Melvill, mais son bord ventral est plus courbé, et l'extrémité postérieure est plus saillante. Outre cela, les deux formes mentionnées semblent être privées de la striation oblique ou divariquée qui marque l'espèce nouvelle et qui n'a pas été décrite pour autre espèce de ce genre.

${ }^{1}$ Moll. of South Africa (Pelecypoda). Marine Investigations in S. Africa, Vol. IV. Cape Town. 
Perna isognomon Linné. Tamatave, 1 ex., long. $82 \mathrm{~mm}$. Cet exemplaire a 9 sillons ligamentaires dans le bord supérieur (dont la largeur est $12 \mathrm{~mm}$ ) et ressemble par sa taille un peu à la $P$.rudis, mais des formes intermédiaires de Mahakamby prouvent qu'il appartient à l'espèce mentionnée.

Ostrea incequivalvis Sowerby. Tamatave, sur le récif de coraux (28/1 1912), 1 ex., long. $53 \mathrm{~mm}$. Correspondant à la fig. 82 de ReEve (Conch. Icon. 18), mais la couleur violette est plus répandue, et n'apparaît pas en forme de raies.

O. radiata VALenciennes. Tamatave, dragage ( $2 / 31912)$, 1 petit ex.

O. cucullata Born. Tamatave, 2 valves, max. long. 87, larg. $55 \mathrm{~mm}$, dont l'une est prolongée en forme de cornet comme montre la fig. $34 a$ de Reeve (Conch. Icon. 18).

Septifer bilocularis Linné. Tamatave, sur le récif de coraux ( $28 / 1$ 1912), plusieurs ex., max. long. $17 \mathrm{~mm}$. La couleur est rouge-foncée à l'extérieur, à l'intérieur il y a sur le fond bleu une teinte assez répandue du même rouge.

S. excisus Wiegmann. Tamatave, sur le récif de coraux 1 ex., long. $24 \mathrm{~mm}$. Cette espèce est caractérisée par la sinuosité large du septum intérieur, qui est courbé au milieu et incisé aux deux extrémités, ainsi que par les côtes épaisses et granulifères. Elle se trouve, d'après LYNGE 1909, au Golfe Persique et dans la mer Rouge jusqu'au Golfe de Siam et à Funafuti.

Modiola philippinarum Hanley. Tamatave, 1 ex., long. $62 \mathrm{~mm}$.

M. auriculata Krauss. Tamatave, sur le récif de coraux (28/1 1912), plusieurs ex., max. long. $53 \mathrm{~mm}$. Comparés avec des ex. de Mauritius (R. M.) ces échantillons font apparaître une sinuosité plus faible dans le bord supérieur ainsi qu'une aile moins prononcée en avant. Peut-être que cette condition dépend de la taille extraordinairement ventrue de la coquille. Les ex. de Mauritius aussi montrent une grande variation en même respect. CH. Hedley $(1909)^{1}$ constate l'espèce de l'Australie.

${ }^{1}$ Moll. from the Hope Islands, North Queensland. Proc. Linn. Soc. N. S. Wales. Vol. 34. 
Mytilus pictus Bonn. Fénérive, sur les rochers de la rivière $(6 / 11912)$, plusieurs ex., max. long. $74 \mathrm{~mm}$, haut. 29,5 , larg. $23,5 \mathrm{~mm}$; un autre ex. mesure: long. 65 , h. 27,5, larg. $19,5 \mathrm{~mm}$. La hauteur, par conséquent, est assez petite relativement à la longueur, et les exemplaires sont fortement allongés. Le fait qu'ils appartiennent à cette espèce, est prouvé par la coloration caractéristique consistant en lignes concentriques en zigzag, qui sont visibles à la partie antéroinférieure. La partie postérieure est verte-olive. Cette espèce n'est pas mentionnée par v. Martens 1880. Elle se trouve à Aden (Sмith 1891) et dans le Golf Persique (Melvill \& StANDEN 1906). ${ }^{1}$

Arca imbricata Bruguière. Tamatave, 4 ex. dans un madrepore sur le récif de coraux $(28 / 1)$, max. long. 17,3 $\mathrm{mm}$ (=A. kraussi Philippi).

A. tenella Reeve. Tamatave, sur la partie du sud-est du récif de coraux (mars 1912), 1 ex., long. $9 \mathrm{~mm}$. Il montre les mêmes caractères que reproduit LyNGE (1909, pl. 1, figs. $11-13\rangle$, la série antérieure des dents très courte et espécialement distincte; dans l'intérieur de la coquille se présentent des stries radiantes surtout en arrière; la couleur est brune claire, rose sur les sommets, avec une large raie d'une couleur rouge-brune entre le milieu de la coquille et la carène; l'intérieur est rouge-brun foncé.

Mytilicardia variegata Bruguière. Tamatave, sur le récif de coraux $(28 / 1), 2$ ex., max. long. $14 \mathrm{~mm}$.

Lucina angulifera v. Martens. Tamatave, dragage, 1 valve gauche, long. $13 \mathrm{~mm}$. Correspondant à la fig. 14, pl. 22, chez v. Martens (1880), qui la cite des Seychelles, de Mauritius et de la mer Rouge.

L. elongata $\mathrm{n}$. sp. Pl. 2, fig. 19. Tamatave, dragage, 1 valve gauche, long. $8,2 \mathrm{~mm}$. Coquille ovale, à crochets médians, allongée au front, largement courbée à l'extrémité, la partie postérieure saillante et plus étroitement arrondie; le bord supérieur plus inclinant en arrière des crochets qu'en avant. Sculpture formée de fines lamelles régulièrement con-

${ }^{1}$ Proc. Zool. Soc. London. 
centriques, 20 en nombre, et de lignes radiantes très serrées sur toute la coquille. Un sillon indistinct s'étend du sommet vers l'arrière. Charnière à une dent cardinale centrale et une dent latérale de chaque côté (valve gauche). Couleur claire jaunâtre.

Tellina ostracea Hanley. Tamatave, dragage ( $2 / 3), 1$ valve gauche, long. $6 \mathrm{~mm}$. Elle a des costules concentriques fines et serrées, lamelliformes en arrière et portant des épines en croissant, postérieurement les 3 côtes rayonnantes des sommets, dont l'antérieure n'est distincte que vers le bord ventral. La coquille est suffisamment identifiée d'après la fig. $124 a$ chez Reeve.

T. pellucida Philippi. Tamatave, dragage, 1 valve gauche, long. 9,2 mm. ReEve a donné une figure (Conch. Icon. 17, fig. 162), qui représente un exemplaire un peu plus haut que cet échantillon. Je doute, si ceci n'appartient pas, au contraire, à la $T$. candidata de Sowerby (Journ. of Conch. 1893) de Durban, puisque la description de cette espèce semble s'accorder bien à l'exemplaire présent. Comme une figure manque, je ne puis l'identifier avec certitude.

T. perna Spengler. Tamatave, dragage $(2 / 3), 3$ fragments concordant à la fig. 12 (Conch. Icon. 17) de ReEve par leur couleur rouge claire avec un rayon blanc en arrière, par leur rostrum épais et sinueux à l'inférieur, et par leur surface complètement lisse (sculptée seulement de stries d'accroissement).

T. rhomboides QuOY \& GAImard. Tamatave, dragage, 1 ex., long. $9 \mathrm{~mm}$, couleur jaunâtre, ressemblant à la fig. $114 a$ chez Reeve (Conch. Icon. 17).

T. semitorta Sownrby. Tamatave, dragage, 1 v. dr., long. $9 \mathrm{~mm}$. Par la taille ces ex. de Madagascar ressemblent à la fig. 221 (Conch. Icon. 17) de Reeve, provenant de Port Jackson, mais les sommets sont un peu plus saillants, derrière les sommets il y a une petite échancrure au contour du bord supérieur, caractère partiellement dépendant de l'absence de ligament des valves présentes. LyNGE a bien figuré (1909, pl. III, figs. 43-46) de nouveau cette espèce qui prouve, 
grâce a son occurrence sur les côtes de Madagascar, une distribution plus vaste que celle constatée jusqu'ici (du Golfe de Siam jusqu'à Torres Strait et N. S. Wales, Lynge 1909).

Syndesmya (Iacra) lactea Dunker. Pl. 2, fig. 20, 21. Tamatave, dragage ( $(2 / 3)$, plusieurs valves, max. long. $12 \mathrm{~mm}$. L'espèce est si caractéristique qu'on la reconnaît aussitôt par sa sculpture divariquée. Probablement, cette forme est identique à Iacra seychellarum A. Apams, espèce qui n'a jamais été figurée. Pour la synonymie je réfère d'ailleurs à LAMY (1913).

Donax madagascariensis WooD. Tamatave, dragage $(2 / 3$ 1912), 1 v. g., long. $9 \mathrm{~mm}$.

D. semisulcatus Hanley. Tamatave, dragage, plusieurs valves, max. long. 9,5 $\mathrm{mm}$. Les exemplaires correspondent aux figs. 1-4, taf. 12, chez Römer (1869). ${ }^{1}$ La sculpture ainsi que la forme sont très variables, celle-ci est souvent relativement haute, comme montrent les fig. de Römer, souvent plus allongée comme chez les ex. figurés par Reeve (Conch. Icon. 8, fig. 56). La sculpture diffère de celle de l'exemplaire de REEve, par les plis moins forts que ceux du dit ex. de Reeve.

D. productus n. sp. Pl. 2, fig. 22, 23. Tamatave, dragage, 2 v. g. + 1 v. dr., max. long. 9,4 mm. Coquille allongée, largement cunéiforme, le bord antéro-supérieur droit, convergeant avec le bord inférieur faiblement convexe; partie antérieure étroitement arrondie, bord postéro-supérieur court et brusquement incliné, formant un angle obtus avec le bord postérieur qui est perpendiculaire et passe par un coin arrondi au bord ventral, dirigé perpendiculairement au bord postérieur. Toute la coquille sculptée de lignes radiantes élevées; surface luisante. Couleur blanche, opaque, avec ou sans quelques rayons rouges, distinctement visibles à l'intérieur. Bord inférieurement crénelé. Largeur max. 2,2, hauteur 5,5 mm.

Mactra lurida Philıpp. Tamatave, dragage $(2 / 3)$ plusieurs valves, max. long. 17 , h. 13 , crassitude (d'une valve) $4,5 \mathrm{~mm}$; une autre valve: long. 14,2 , haut. 12 , crass. $4,3 \mathrm{~mm}$. L'espèce

1 Die Fam. Donacidæ, Martini \& Chemnitz, Conch. Cab. 
varie donc considérablement concernant la forme; aussi la sculpture est un peu variable, comme la première espèce est presque tout à fait lisse, n'ayant que la lunule et le corselet régulièrement costulés, pendant que ces costules continuent comme des rides très obscures sur les côtés de la coquille; chez l'autre espèce, au contraire, les costules sont plus distinctes, et par-dessus cela, une carène anguleuse s'étend des sommets en arrière limitant le corselet. La couleur est isabelle claire tirant dans le lilas et le violet vers les sommets; les rayons sont très indistincts; mais tels se trouvent chez des autres échantillons. WEInkaufF (1884) et Lynge (1909) considèrent $M$. lurida comme se rapprochant de $M$. corbuloides Deshayes, mais cette espèce a une forme plus triangulaire; en outre, la forme de Madagascar a un sinus palléal plus profond que le montre la fig. 18 tabl. IV chez Lynge, représentant $M$. cuneata Chemnitz (=M. corbuloides). - De Mozambique se trouvent, dans les collections du R. M., des ex. qui ne diffèrent de la forme présente que par leur bord postérieur plus courbé.

M. opposita Deshayes. Tamatave ( $7 / 2), 2$ ex., max. long. $12 \mathrm{~mm}$. La forme est hautement triangulaire à petits sommets, comme montre la fig. 11 de ReEve (Conch. Icon. 8), mais comme les ex. sont usés et la couleur en est absente, l'identité n'est pas certaine; la lunule et le corselet sont tachés de brun comme dans la fig. 95 chez Reeve (de $M$. opposita), mais le violet des sommets de la figure n'est pas visible.

Cytherea hebrcea Lamarck. Tamatave, dragage ( $2 / 3$ 1912), 2 ex., max. long. 7,5 mm, comparés et pareils à des ex. de la mer Rouge (coll. M'Andrew). L'espèce n'est pas citée par v. Martens (1880).

C. ambigua Deshayes. Tamatave, dragage ( $2 / 3$ 1912), 4 ex., long. $8 \mathrm{~mm}$. Bien que petits, les exemplaires montrent les caractères typiques: une forme étendue triangulaire, de larges rayons brun-violets, les bords dorsaux bruns et par tout cela ils s'accordent avec la fig. 37 chez Reeve (Conch. Icon. 14, Cytherea). 
C. arabica Chemnitz. Tamatave, dragage, $1 \mathrm{v} . \mathrm{g} .+1 \mathrm{v}$. dr., max. long. $9 \mathrm{~mm}$. La forme et la sculpture s'accordent avec celles des spécimens de Savigny (Descr. de l'Egypte, pl. 9, fig. 6), qui, selon ISSEL (1869), sont l'espèce présente.

C. striata Gray. Tamatave, dragage ( $2 / 31912), 2$ v. dr., max. long. $8 \mathrm{~mm}$. Par son extrémité postérieure subcostulée et saillante et par le contour supéro-postérieur fortement arrondi, cette valve semble être la même espèce que celle figurée par ReEve (Conch. Icon. 14, Dione, fig. 44).

Tivela polita Sowerby. Fénérive (6/1), 3 ex., max. long. $5,7 \mathrm{~mm}$.

Chione marica Linné. Tamatave, dragage ( $2 / 3$ 1912), plusieurs ex., max. long. $11 \mathrm{~mm}$. Ils diffèrent de la forme typique (voir LyNGE 1909, pl. V, fig. 1-4) par l'absence de squamelles à la partie postérieure de la coquille, où les lamelles concentriques diminuent par degrés en force vers le bord supérieur. Il y a, au contraire, un caractère typique dans la présence, au corselet, de costules radiantes qui diminuent successivement en force relativement aux autres de la coquille. Par ce caractère l'espèce se distingue de Ch. recognita E. A. Smith (1885), ${ }^{1}$ qui a le corselet lisse, et de Ch. costellifera Adams \& ReEve (Conch. Icon. 14, fig. 103), qui a le même finement strié (les stries plus serrées et plus fines que les autres de la coquille, selon des ex. de la coll. M'AndRew au R. M.). La taille de la forme de Madagascar est relativement élevée (= la fig. de Ch. recognita chez Sмiтн, pl. III, fig. 5), sans aucune trace de rostrum, ce qui se trouve chez Ch. costellifera; par la couleur elle ressemble en partie à la fig. $104 a$ de REEve (de Ch. marica) mais de l'autre côté elle est presque unicolore et moins tachetée.

Tridacna elongata Lamarck. Fénérive, 1912, 3 ex., max. long. $120 \mathrm{~mm}$. - Tamatave, sur le récif de coraux ( $\left.{ }^{4} / 21912\right)$, 2 ex., max. long. $70 \mathrm{~mm}$.

Chama spinosa Broderip. Pl. 3, fig. 35-39. Tamatave, sur la partie du sud-est du récif de coraux, plusieurs ex., attachés aux coraux, max. diam. (sommet-bord ventral) 18

${ }^{1}$ Rep. Challenger. Lamellibranchiata. 
mm. - Ces exemplaires sont couverts d'une croûte calcaire qui masque pour la plupart la sculpture caractéristique d'épines serrées qui apparaît, cependant, clairement dans un individu jeune. A l'intérieur les valves montrent une teinte claire verdâtre pendant que les dents principales et souvent le bord supérieur sont plus ou moins colorés de violet. Le bord intérieur crénelé ou lisse. L'espèce qui est attachée par sa moitié antéro-supérieure varie beaucoup en forme, comme l'indiquent les figures. La valve inférieure est fortement enroulée, ce qui est typique pour l'espèce.

Saxicava rugosa Linné. Tamatave $(5 / 3)$, sur le récif de coraux, 1 ex., long. $6 \mathrm{~mm}$.

?Cultellus attenuatus Dunker. Pl. 2, fig. 14. Tamatave, dragage $(2 / 31912)$, une petite valve droite, $10 \mathrm{~mm}$ de longueur. Chez l'ex. figuré par Reeve (Conch. Icon. 19, fig. 8), la partie postérieure de la coquille est plus allongée que celle-ci et la détermination est incertaine, comme je n'ai pas à ma disposition les matériaux nécessaires pour établir une comparaison.

Patella pica Reeve. Tamatave, sur le récif de coraux, 4 ex., max. long. $57 \mathrm{~mm}\left({ }^{28} / 1\right.$ 1912); Fénérive, les rochers $(6 / 1)$, plusieurs ex., max. long. $50 \mathrm{~mm}$.

Helcioniscus rota Gmelin. Tamatave, sur le récif de coraux, 4 ex., max. long. $24 \mathrm{~mm}$. - Fénérive, les rochers ( $6 / 1$ 1912), plusieurs ex., max. long. $19 \mathrm{~mm}$. La sole du pied des animaux est grise-noire, et par cela ils se distinguent des animaux uniformement blanes de Patella pica.

Phasianella zigzag n. sp. Pl. 2, fig. 25. Coquille largement conique, à tours convexes. Couleur: $3-4$ séries de taches, alternant des brunes et des blanches, enceignent le dernier tour; les taches brunes sagittiformes et liées obliquement entre elles par des lignes brunes qui forment des fulgurations longitudinales à travers les tours, comme chez $P h$. speciosa. Les séries spirales de taches souvent indistinctes, mais les lignes fulgurantes très constantes et caractéristiques. Dimensions: hauteur 4. largeur 2,6 $\mathrm{mm}$; nombre de tours 5 . Localité: Fénérive, les rochers à mer $(6 / 1), 2$ ex. 
Minolia variegata n. sp. Pl. 2, fig. 26-28. Coquille déprimée trochiforme à spire courte proéminente et les tours aplatis aux sutures, profondement et largement ombiliquée; ouverture arrondie avec le bord columellaire un peu sinueux, et le bord extérieur bien courbé. Sculpture composée de fortes carènes spirales, séparées par des sillons excavés au nombre de 7 au-dessus du milieu du dernier tour et 3 immédiatement en bas; surface basale lisse sauf de fines traces à stries. Dans l'ombilic 4 lignes spirales divisées en tubercules. Couleur claire jaune-brunâtre (parfois grise-brune) avec des groupes de petites taches brunes arrangées en spiral et plus ou moins irrégulières, parfois confluents longitudinalement, et parfois fusés à un ton assez foncé; le côté basal constamment avec une série périphérique de taches et, en dedans, une bande jaune, olive-brune ou olive-verte; l'intérieur de l'ombilic blanc-pur. Dimensions: haut. $7 \mathrm{~mm}$; larg. 8 ; h. du dernier tour 4 ; tours $61 / 2$; un autre ex.: haut. 6,2 ; larg. 8,2 ; h. du dernier tour 3,5 , tours $61 / 2$. Quand la hauteur est grande, l'ombilic devient plus étroit; à grande largeur, l'ombilic est plus ample. - Tamatave, dragage, plusieurs ex.

Cette espèce ressemble un peu en couleur et en sculpture à la $M$. ornatissima décrite par Schepman (1908, Siboga Exp.), qui a, cependant, le dernier tour anguleux et la base striée.

Trochus mauritianus Gmelin. Tamatave, sur le récif de coraux, 5 ex., max. haut. 58 , larg. $49 \mathrm{~mm}$; un autre ex.: h. 56, larg. $53 \mathrm{~mm}$. Ils s'accordent par leurs cordes spirales basales avec des ex. typiques (voir Tryon 1889, Man. of Conch. 11, pl. 4, fig. 24, 25).

Clanculus puniceus PhilipPi. Tamatave, sur le récif de coraux, 1 ex., diam. $20 \mathrm{~mm}$.

Turbo argyrostomus Linné. Tamatave, sur le récif de coraux, 1 ex., haut. $85 \mathrm{~mm}$.

Fissurella mutabilis Sowerby. Fénérive, les rocbers $(6 / 1$ 1912), 2 ex., max. long. $18 \mathrm{~mm}$. Ces ex. sont caractérisés par 6-7 fascicules à rayons rouges de chaque côté, des costules radiantes indistinctes $(3-4$ rouges dans chaque fascicule, les interstices blanches), et par des stries d'accroissement irrégulières, par la coquille compacte et un foramen allongé, 
aux côtés à peine sinueux. Par ces caractères ils s'accordent bien à la fig. $43 a$ de Reeve (Conch. Icon. 6). Une distinction spécialement spécifique est le fait qu'un rayon blanc s'étend du milieu du côté de la perforation (au contraire de la $F$. nubecula de la Méditerranée, où cette place est occupée par un rayon rouge).

Neritina rangiana Recluz. Tamatave, 1 petite coquille, long. $4 \mathrm{~mm}$; une série de taches blanches suturales et la couleur verte la caractérisent.

Natica marochiensis GmeLiN. Fénérive, 2 ex., max. haut. $8 \mathrm{~mm}$. Ils sont colorés de 2 larges bandes olive-vertes grisâtres, la supérieure, plus large, flanquée par des taches brunes, l'inférieure traversée par de pareilles en avant, tandis que les taches, en arrière, se séparent en deux séries: la supérieure forme une raie de taches et l'inférieure se fuse à une bande. Le tour de l'ombilic ainsi qu'une bande suturale sont blanches.

N. picta Recluz. Tamatave, 1 ex., haut. $17 \mathrm{~mm}$. L'exemplaire s'accorde par sa forme et son dessin à la fig. $67 c$ de Reeve (Conch. Icon. 9), mais il en diffère par sa couleur plus claire. Il est d'un blanc luisant et il porte 2 bandes spirales brune-claires, l'une au-dessus et l'autre au-dessous du milieu du dernier tour, et une indication d'une troisième bande près de la suture; les zones intermédiaires sont blanches et traversées par des lignes brunes sagittées comme chez le type. Pour cette raison je le réfère à l'espèce dite comme un exemplaire pas encore adulte. De l'autre côté il ressemble à $N$. maheensis Recluz (Reeve, l. c. fig. 58), par les sillons obliques qui partent de la suture, mais l'ombilic est différent de celui de l'autre espèce. $N$. picta n'a pas été citée par v. Martens 1880. Elle est distribuée entre les Philippines, l'Australie du Sud et la Nouvelle-Calédonie (Tryon).

N. trailli Reeve. Tamatave, 1 coquille, haut. $3,8 \mathrm{~mm}$, avec des stries transversales brunes serpentantes et un cal brun comblant l'ombilic, comme dans la fig. de Reeve (Conch. Icon. 9, pl. XXIX, fig. 137).

$N$. asellus Reeve. Tamatave, 1 coquille, haut. $6 \mathrm{~mm}$. Deux bandes bleues-brunes et une série de taches entre elles; 
les bandes montrent la particularité d'être traversées par des stries brunes en même nombre que les taches intermédiaires et radiantes en direction aux taches.

N. ala papilionis Chemnitz. Tamatave, dragage, 2 ex., max. haut. $6,5 \mathrm{~mm}$, à couleur très pâle mais à dessin typique.

C. mamilla Linné. Tamatave, dragage, 4 ex., max. long. $16 \mathrm{~mm}$.

Sigaretus papilla Gmelin. Tamatave, dragage, 1 ex., haut. $13 \mathrm{~mm}$. L'espèce n'a pas été citée par v. Martens 1880 .

Hipponyx australis QUOY \& GaIMARD. Tamatave, sur le récif de coraux, 3 ex., fixés sur Turbo argyrostomus, max. long. $15 \mathrm{~mm}$.

Cerithium kochii Philippi. Tamatave, dragage ( $2 / 3$ ), 4 ex., max. long. $26 \mathrm{~mm}$. Connu auparavant, selon v. Martens, de la mer Rouge, des Indes et de Mauritius.

Bittium amboynense Watson. Fénérive, les rochers, 1 ex., long. $3,7 \mathrm{~mm}$. Le fil spiral épais, caractéristique pour l'espèce et situé au-dessous de la suture et sur le milieu du dernier tour est distinctement saillant, et la sculpture ressemble du reste aussi à la fig. de Watson (Challenger Rep., t. 140, fig. 5). La base est, cependant, sculptée de la même façon que l'autre côté de la coquille, par des cordelettes spirales, mais non par des tubercules. Il y en a 4 séries au dernier et 3 aux tours supérieurs. La couleur est brunefoncée.

Littorina glabrata PhilippI. Tamatave, au quai, plusieurs ex., max. haut. $17 \mathrm{~mm}$.

Pterocera chiragra Linné. Tamatave, sur le récif de coraux (Mars 1912), 1 ex., long. $160 \mathrm{~mm}$; sur la coquille 2 ex. de Vermetus sp.

P. bryonia Gmelin. Tamatave, sur le récif de coraux, 3 ex., max. long. $360 \mathrm{~mm}$. 
Strombus canarium Linné. Tamatave, dragage, 20-25 mm $(2 / 3), 2$ ex., pas encore adultes, max. haut. $30 \mathrm{~mm}$.

S. labiosus Gray. Tamatave, sur le récif de coraux $\left({ }^{4} / 9\right)$ 1 ex., haut. $53 \mathrm{~mm}$. L'exemplaire s'accorde complètement par sa couleur et par sa forme à la fig. 50 de Reeve (Conch. Icon. 6). La couleur en est jaune-brune sur les tours, blanche dans l'ouverture et sur le bord extérieur épais; la sinuosité de ce bord est peu profonde. Il en diffère par ses costules longitudinales plus épaisses et espacées sur le dernier tour près de l'ouverture, ainsi que par ses tours supérieurs plus bas et plus larges (9 en nombre sauf le dernier). De $S$. columba il diffère par la sinuosité du bord extérieur moins profonde, et par le fait que le sinus supérieur de l'ouverture n'est pas étendu en haut sur la spire. L'espèce n'était pas connue auparavant de Madagascar (Philippines et Australie sont cités comme ses habitats par Tryon 1885).

Cirsotrema soror n. sp. Pl. 3, fig. 31-33. Coquille allongée pyramidale, pointue, à tours convexes, et à sutures assez profondes; l'ombilic couvert. Sculpture composée d'épaisses costules, longitudinales, serrées, ca. 15 sur un tour. Une carène basale limite les costules à la partie inférieure du dernier tour. L'ouverture obliquement ovale, le péristome continu, labre extérieur épais. Coquille couverte d'un épiderme assez épais, calcaire, sculpté de fines lignes spirales et longitudinales se croisant et donnant l'aspect d'un tissu subtile. Dimensions: long. 14,4; larg. 4,6; long. de l'ouverture $3,5 \mathrm{~mm}$. Couleur blanche-opaque. Localité: Tamatave, dragage $(2 / 3), 1$ ex.

Cette espèce ressemble à $C$. texta E. A. Sмiтн 1903, de l'Archipel des Maldives, mais celle-ci a des sillons spiraux sur toute la coquille, qui sont absents chez la forme présente.

Eulima algoënsis Sмiтн 1901. Tamatave, sur le récif de coraux, 1 ex., long. $6 \mathrm{~mm}$. Bien qu'un peu plus petit que l'exemplaire de Sмıтн, qui a le sommet rompu et qui dérive de l'Algoa Bay, l'individu présent ressemble par ses proportions tout à fait à la fig. de Sмгтн (les 2 derniers tours ensembles sont de la même longueur que les supérieurs, et tous sont faiblement convexes). Le nombre des tours est 12, et le sommet est fléchi à droite. La forme présente a, cepen- 
dant, un dernier tour un peu plus épais et l'ouverture plus étroite. Il ressemble par cela à $E$. munda SMITH (Journ. of Conch. vol. IX, 1899, p. 248, pl. V, fig. 7), mais chez cette espèce l'ouverture est plus haute ( $>$ le diamètre du dernier tour à la suture).

Dolium costatum Menke. Tamatave, dragage, 1 petit ex., haut. $9 \mathrm{~mm}$.

D. perdix Linné. Angotsy, Tamatave, 1 ex., haut. $140 \mathrm{~mm}$.

Bullia belangeri Kiener. Fénérive ( $6 / 1), 3$ ex., max. haut. $16 \mathrm{~mm}$. Forme et sculpture (les stries spirales plus distinctes près de la suture et sur la partie basale) ainsi que la couleur (des flammes et des raies jaunes longitudinales) sont des earactères communs à la fig. donnée par ReEve (Conch. Icon. 3, fig. 8).

Nassa coronata Bruguik̀re. Tamatave, dragage ( $2 / 31912)$, plusieurs ex., max. haut. $12 \mathrm{~mm}$.

$N$. arcularia Lamarck. Tamatave, 1 petit ex., long. 25 $\mathrm{mm}$.

$N$. kieneri Deshayes. Tamatave, 1 petit ex., correspondant aux ex. typiques des collections du R. M.

Cyprcea carneola Linné. Tamatave. 1 coquille, à couleur caractéristique, long. $44 \mathrm{~mm}$.

C. caurica Linné. Tamatave, 1 jeune ex., un peu usé.

C. arabica Linné. Tamatave, 1 ex, long. $72 \mathrm{~mm}$, montrant une coloration consistant de nuages bruns et de deux bandes transversales plus claires; au-dessous de la suture des lignes brunes radiantes; la couleur est par conséquent celle d'un jeune ex.

C. annulus Linné. Tamatave, sur le récif de coraux (28/1 1912), plusieurs ex., max. long. $27 \mathrm{~mm}$.

C. caput serpentis Linné. Tamatave, sur le récif de coraux (28/1, 1912), 1 ex., long. $27 \mathrm{~mm}$. 
C. hebrcea Lamarck. Tamatave, dragage, 1 ex., long. $5,2 \mathrm{~mm}$.

Ovula insculpta n. sp. Pl. 3, fig. 34. Coquille ovale, aux extrémités également prolongées, assez gonflée au milieu. Surface polie, sculptée de fines lignes transversales, impressées et ponctuées à des intervalles réguliers (chaque intervalle a une largeur d'environ trois stries). Couleur claire rouge grisâtre, les extrémités et le labre extérieur blancs; une zone grisâtre traversant le milieu de la coquille. Bord extérieur épais, un peu réfléchi, presque lisse à l'intérieur (de faibles traces de dents présentes), avec un sinus mince à l'extrémité inférieure. Columelle avec un pli supérieur et une impression flanquée d'un cal dans le bas. Ouverture étroite, élargie à l'inférieur, contractée près des canaux des deux extrémités. Dimensions: long. 16, larg. $7 \mathrm{~mm}$. - Localité: Majunga, dragage, 1 ex.

Cette espèce ressemble par sa forme à l'O. sowerbyana Weinkauff (Tryon, Man. of Conch. 7, pl. 5, fig. 26, 27), mais elle est sculptée à la façon de l'O. borbonica Deshayes (1863, p. 136; pl. XIII, fig. 18-20), une espèce qui est, cependant, différente par sa taille plus petite et ses extrémités moins allongées.

Mitra flammea Quoy \& GaImard. Tamatave, dragage, 2 ex., max. long. $15 \mathrm{~mm}$, semblables à la fig. 191, pl. 41 de Tryon (Man. of Conch. 4, 1882) mais différents de la fig. 120 de Reeve (Conch. Icon. 2), qui est identique à la M. philippinarum A. Adams selon Tryon.

M. (Turricula) interpunctata n. sp. Pl. 3, fig. 29. Coquille fusiforme, la spire ainsi que l'ouverture allongée et à peu près de la même longueur. Sculpture composée de costules longitudinales serrées, plus distinctes sur les tours supérieurs qu'au dernier (nombre 36 au dernier tour); dans les interstices des points en séries régulières spirales (14 sur le dernier tour), à la partie basale 4 sillons creusés entre les 5 plis columellaires. L'ouverture étroite d'une largeur uniforme, en haut avec un cal faible; la parois extérieure cannelée (en dedans); le bord extérieur simple, canal distinctement limité, court, étroit. Sommet $\left(2^{1 / 2}\right.$ tours) lisse, les 3 tours suivants portant des costules longitudinales et une série de tubercules 
près de la suture. Le nombre des tours 9. Couleur jaune brunâtre avec une teinte plus claire formant une série de taches carrées blanches (alternant avec des brunes) sur chaque tour au-dessus du milieu du dernier. Canal un peu plus clair que les taches brunes. Ouverture blanche opaque en dedans. Dimensions: long. 10,5, larg. 3,8, hauteur de l'ouverture 5,7 mm. Localité: Tamatave, dragage, 1 ex. ( $2 / 3$ 1912).

Turricula (Costellaria) exasperata Gmenin. Tamatave, dragage, 6 ex., max. haut. $25 \mathrm{~mm}$; s'accorde avec les fig. 544 -546 (pl. 53-54) de Tryon (Man. of Conch. 4, 1882).

Rapa papyracea Lamarck. Tamatave, sur le récif de coraux ( $2 / 3$ 1912), 2 ex., baut. $27 \mathrm{~mm}$; s'accorde avec la fig. de Tryon (Man. of Conch. 2, pl. 67, fig. 392) et avec les ex. typiques des coll. du R. M.

Rhizochilus madreporarum Sowerby. Tamatave, sur le récif de coraux $\left({ }^{4} / 2\right.$ 1912), 1 ex., long. $10,2 \mathrm{~mm}$.

Oliva tigrina Lamarck. Tamatave, dragage, plusieurs ex., max. long. $20 \mathrm{~mm}$.

Ricinula ochrostoma Blainville. Tamatave, sur le récif de coraux $(4 / 2), 1$ ex., haut. $32 \mathrm{~mm}$.

Cancellaria costifera Sowerby. Tamatave, l ex., haut. $11 \mathrm{~mm}$, montrant des lignes spirales rouges interrompues, distinctes seulement à l'arrière des côtes: 7 plis longitudinaux dans un demi-tour (vu de l'ouverture); couleur brune-claire bleuâtre.

Nassaria acuminata Reeve. Tamatave, dragage, 1 ex., haut. $22 \mathrm{~mm}$, s'accordant avec la fig. de Triton acuminatus de Reeve, Conch. Icon. 2, pl. XIV, fig. 54, et avec celle de Tryon, 1881, Man. of Conch. 3, pl. 84, fig. 539, 540.

Terebra duplicata Linné. Tamatave, dragage, 2 ex., max. haut. $25 \mathrm{~mm}$, ressemblant à la fig. $3 a$ de Reeve (Conch. Icon. 12), par une étroite ligne brune au milieu du dernier tour.

T. strigilata Linné. Tamatave, dragage, 2 ex., max. long. $22 \mathrm{~mm}$, s'accordant avec la fig. 84, pl. 10, de Tryon (Man. 
of Conch. 7, 1885). - Fénérive, ( $6 / 1$ 1912), 1 ex., long. 10,5 $\mathrm{mm}$. La taille plus élancée le différencie des ex. précédents, mais la sculpture et la couleur s'accordent avec eux concernant les tours supérieurs.

T. straminea Gray. Tamatave, 2 coquilles, max. haut. $28,5 \mathrm{~mm}$, tours 19 , couleur brune foncée. L'espèce n'est pas citée par v. Martens (1880).

T. polygonia Reeve. Pl. 3, fig. 38. Tamatave, dragage, 2 ex., max. long. 14,5 mm. Les deux ex. s'accordent en couleur et en sculpture à la figure donnée par REeve (Conch. Icon. 12, fig. 154). La couleur est jaune-brune grisâtre avec un lustre soyeux, le sillon sutural est distinctement marqué comme une ligne claire interrompue de plis et entouré d'une zone indistinctement plus foncée, une bande subsuturale d'une couleur un peu plus claire que le ton fondamental; les plis sont 6-7 au dernier demi-tour. L'exemplaire de ReEve est d'un habitat inconnu, c'est donc un fait des plus intéressants que de savoir que cette espèce se trouve à Madagascar, d'autant plus, qu'elle ne semble pas avoir été signalée depuis qu'elle fut décrite par Reeve.

Conus vexillum Linné. Tamatave, 1 ex., haut. $115 \mathrm{~mm}$.

C. tulipa Linné. Tamatave, 1 ex., long. $80 \mathrm{~mm}$.

C. hebrceus Linné. Tamatave, 1 ex., long. $45 \mathrm{~mm}$.

Actcoon flammeus Gmelin. Tamatave, 1 ex., long. $7 \mathrm{~mm}$, s'accorde par sa couleur aux fig. 58, 59, pl. 20 A, de Pilsbry (Man. of Conch., Vol. 15, 1893), bien que les 2 zones supérieures de taches soient plus espacées. Sculpture sillonnée, ponctuée.

Cylichna strigella A. Adams. Tamatave, 1 coquille, haut. $8,5 \mathrm{~mm}$, probablement appartenant à cette espèce à en juger par la fig. de Pilsbry (Man. of Conch., Vol. 15, 1893, pl. 48, fig. 14). L'espèce était connue auparavant des Philippines et de Torres Straits; aucune espèce de ce genre n'est mentionnée de Madagascar.

Asteronotus mabilla Bergh. Tamatave ( $4 / 2$ 1912), sur le récif de coraux, 1 ex., long. $70 \mathrm{~mm}$. 
Sphcerodoris lovis Bergh. Tamatave ( $4 / 2$ 1912), sur le récif de coraux, 1 ex., long. $22 \mathrm{~mm}$.

Onithochiton lyelli Sowerby. Tamatave $(4 / 21912), 1$ ex., long. $10 \mathrm{~mm}$. L'exemplaire s'accorde complètement avec la description et les figures données par Pilsbry (Man. of Concl. 14, 1892 , p. 247 , pl. 55, fig. 1-7). Auparavant, l'espèce n'était connue que de l'Australie.

Acanthochites penicillatus Deshayes. Tamatave, sur le récif de coraux $(28 / 11912), 3$ ex., max. long. 9,5, larg. $5 \mathrm{~mm}$. Quoique ces exemplaires ne soient pas tout à fait typiques, je n'hésite pas à les référer à l'espèce nommée. La différence semble être trop mince pour justifier la création d'une nouvelle espèce. Ce n'est que la forme de la dernière valve qui est un peu atypique, puisque son tegmentum n'est pas circulaire (comme montre la fig. 11, pl. VI de Thiele, 1909), mais élargi, et puisque la valve entière a une largeur deux fois plus grande que la longueur. Ia sculpture des valves se compose de gros granules ovales, pas en forme de gouttes comme chez le type, mais cette taille des granules a été rencontrée aussi chez des exemplaires de la mer Rouge, d'après Thiele (1909). Les spicules de la zone ont la même configuration que celle décrite et figurée par Thiele (fig. 12).

Choneplax indicus n. sp. Pl. 3, fig. 44, 45. Corps allongé, vermiforme; valves imbriquées, ovales, aux sommets fortement pointus en arrière; tegmentum divisé en une aire médiane un peu élevée et deux parties latérales séparées de cette première par un sillon bien marqué. Sculpture des valves: aire médiane finement granulée, les granules arrangés en séries longitudinales; aires latérales à granules plus grands irrégulièrement épars. Couleur des valves jaune à taches brunes, plus prononcées aux côtés des aires latérales; à l'intérieur la même couleur laisse apercevoir une teinte rose.

Valve antérieure à surface indivisée et à 3 incisures dans le bord de l'articulamentum et des traces à une ultérieure de chaque côté plus en arrière. Valves intermédiaires sans aucune incisure.

1 Revision des Systems der Chitonen, I, Zoologica, Stuttgart. 
Zone large, claire jaune-verdâtre, revêtue de spicules courtes, hyalines à sommets bruns et verts, entremêlées de grandes isolées; bord garni d'une frange de spicules assez longues. Entre les valves des groupes de spicules un peu plus longues, minces et courbées, lisses aux extrémités obtusées; devant la première valve 4 petits groupes de cette sorte. Le côté inférieur de la zone est revêtu de spicules plus courtes.

Dimensions: Longueur 10, largeur 3, hauteur $2 \mathrm{~mm}$.

Branchies occupant le tiers postérieur de la rainure parapodiale.

Localité: Tamatave, sur le récif de coraux (28/1 1912), $1 \mathrm{ex}$.

Le genre Choneplax compte jusqu'ici deux espèces, l'une, Ch. latus, des Indes occidentales, et l'autre, Ch. hastatus, de localité incertaine (voir Prlssbr, Man. of Conch., Vol. 15, 1893). L'espèce nouvelle diffère distinctement des deux. Ch. latus a des aires dorsales lisses aux valves intermédiaires, et Ch. hastatus porte des touffes suturales de très longues spicules aiguës.

C'est un fait très intéressant qu'une espèce nouvelle de ce genre, qui est connu avec certitude seulement des Indes occidentales, se trouve à Madagascar et dans la région de l'Océan Indien, l'habitat de plusieurs espèces singulières d'Acanthochites (par ex. A. aberrans décrit plus haut) et de Cryptoplax. Choneplax est à considérer comme un chaînon joignant ces deux genres (voir PiLSBRy 1893 et Thiele 1909). Peut-être est-il possible, que les Cryptoplacidés descendent de formes de ces régions et que des recherches plus complètes sur la faune marine de Madagascar révéleront des formes nouvelles de transition combinant les deux familles des Acanthochitidae et des Cryptoplacidae.

\section{Mollusques marins sans localité déterminée.}

Quelques espèces étaient collectionnées sans être pourvues de localités déterminées. Ce sont: Chama brassica Revve, 1 coquille, haut. $85 \mathrm{~mm}$; Gastrochona apertissima Deshayes, plusieurs ex., fourrés dans la coquille de l'espèce précédente, max. long. 17,5 mm; Bulla ampulla Linné, 2 coquilles, max. haut. $31 \mathrm{~mm}$. 


\section{Mollusques marins collectionnés par M. K. Afzelius.}

Les sous-dites espèces dérivent principalement des récifs de coraux de Tuléar, sur la côte du sud-ouest de Madagascar.

Natica melanostoma Gmelin. Borso Bé (12/4 1912), 1 ex., long. $30 \mathrm{~mm}$.

Turbo imperialis Gmelin, var. regenfussi Deshayes. 2 ex., max. haut. 88, diam. $83 \mathrm{~mm}$, avec quelques ex. de Hipponyx australis QuOY \& GAImaRD, max. long. $12 \mathrm{~mm}$. Cette variété diffère de la forme typique par la présence d'une série de tubercules sur le dos du dernier tour. Cette série est chez le plus grand de ces exemplaires plus marquée que chez le petit et, par cela, ne forme pas de caractère spécifique. Les ex. s'accordent avec la fig. de Tryon 1888 (Man. of Conch., Vol. X, pl. 48, fig. 40).

Rapa papyracea Lamarck. 1 ex., haut. $45 \mathrm{~mm}$. L'exemplaire montre la particularité qu'un nouveau siphon s'est formé à trois reprises pendant l'accroissement plus avancée; chacun de ces siphons accessoires se trouve en dedans du précédent et immédiatement au-dessus de son extrémité.

Conus geographus Linné. 1 ex., long. $85 \mathrm{~mm}$.

C. tulipa Linné. 1 ex., long. $67 \mathrm{~mm}$.

Fasciolaria trapezium Linné. 1 ex., long. $130 \mathrm{~mm}$.

Hipponyx australis QUOY \& GaIMARD. Quelques ex. sur Conus tulipa et Turbo imperialis.

Pterocera scorpio Jinné. 1 ex., long. $130 \mathrm{~mm}$.

Pteria margaritifera Linné. 1 ex., long. $100 \mathrm{~mm}$.

Tridacna elongala Lamarck. 2 ex., max. long. $185 \mathrm{~mm}$.

Acanthopleura spiniger Sowerby. 1 ex., long. ca. $60 \mathrm{~mm}$.

Oncidium peroni Cuvier. 3 ex., max. long. $70 \mathrm{~mm}$, montrant des caractères typiques: des tubercules dispersés sur toute la surface dorsale (comparer $O$. tonganum QUOY \& 
Gaimard, qui est identique à l'espèce de Cuvier selon E. v. MaRTENS 1897). ${ }^{1}$

\section{Mollusques aquatiques et terrestres.}

Neritina pulligera Linné, var. knorri Recluz. Androhibé, dans la rivière (Sept. 1912), plusieurs ex., max. long. $29 \mathrm{~mm}$.

Melanatria spinosa Lamarck. Tamatave (Févr. 1912), 2 ex., max. haut. $67 \mathrm{~mm}$.

Melania cybele Gould. Androhibé, dans la rivière (Sept. 1912), 4 ex., vivants, max. haut. $51 \mathrm{~mm}$. Toutes les coquilles étaient incrustées de chaux, cependant un peu moins du côté de l'ouverture. Les exemplaires s'accordent par leur taille allongée, par leurs tours assez élevés et enfin par la solidité de leurs coquilles le mieux avec $M$. cybele Вrot (1874, fig. $1 a$ et $1 b, \mathrm{pl} .30)^{2}$ et diffèrent en même temps de $M$. amarula, qui est commune à Madagascar. L'espèce ne semble pas avoir été mentionnée auparavant de Madagascar. BRot (1874) la signale de Sumatra, des Philippines et des îles de Fiji.

Ampullaria madagascariensis E. A. Sмiтн. Mangabe (Févr. 1912), 1 ex., avec animal, haut. 40 , diam. $37 \mathrm{~mm}$.

Helicophanta magnifica Férussac. Fito, 1 coquille, diam. $55 \mathrm{~mm}$.

H. oviformis Grateloup. S:te Marie de Marovoay (Juin 1912), 2 coquilles, max. haut. $62 \mathrm{~mm}$.

Eulota similaris Férussac. Tamatave ( $20 / 11912)$, plusieurs ex., max. diam. $14 \mathrm{~mm}$. - Fénérive ( $\% / 11912)$, parmi des feuilles pourrissantes, 4 ex., max. diam. $11 \mathrm{~mm}$; $29 / 12,1$ ex., diam. $8 \mathrm{~mm}$.

Nanina (Xesta) hova n. sp. Pl. 4, fig. 50-52, coquille déprimée hélicoïde, la spire convexe et le dernier tour bien

1 M. Weber, Süss- u. Brackwasser-Moll. d. Ind. Archipels. Zool. Ergebnisse, Reise in Niederländ. Ost-Indien.

${ }^{2}$ Melaniaceen, Martini \& Chemnitz, Conch. Cab. 
arrondi à la périphérie, sans trace d'angularité; suture peu imprimée. Surface lisse et luisante, sculptée seulement de très fines lignes d'accroissement un peu irrégulières et de stries spirales microscopiques, les plus distinctes à la base de la coquille. Ombilic étroit, demi-couvert par un plissement de l'extrémité supérieure du bord columellaire et rempli de cal en dedans. Ouverture largement semi-lunaire; péristome simple à labres bien courbés et joints par une couche calcaire très mince sur la paroi interne. Couleur brune de différentes nuances chez divers échantillons (de café ou de corne), un peu plus claire à la base; une bande périphérique fauve entoure le dernier tour et marque, comme une ligne étroite, la suture. Dimensions: haut. 15, diam. max. 25, diam. min. 23, haut. de l'ouverture 11, largeur de l'ouverture 13, diam. de l'ombilic $1,5 \mathrm{~mm}$. Tours 6 .

Localité: Catsèpe, Mai 1912, 3 coquilles.

Les espèces malgaches du genre Nanina ont été mises ensemble par H. DoHrN (1882). ${ }^{1}$ L'espèce présente se rapproche de $N$.ekongensis Angas et de $N$. fuscolutea Grateloupe, mais elle diffère de toutes les deux par sa couleur entièrement brune avec une bande fauve périphérique. Comme les autres espèces malgaches de ce genre sont carénées, elle constitue avec certitude une forme nouvelle.

Achatina fulica Férussac. Fénérive ( $1 / 121911)$, plusieurs ex., max. long. $105 \mathrm{~mm}$. - Mayotte, 3 coquilles, max. long. $70 \mathrm{~mm}$. Un ex. fut envoyé par M. KAUDERN à l'état vivant à Stockholm, ou il vivait pendant deux mois en automne 1912.

Subulina octona Bruguik̀re. Fénérive, parmi des feuilles pourrissantes ( $9 / 1$ 1912), plusieurs ex., max. long. $14 \mathrm{~mm}$.

Urocyclus madagascariensis PoIrIer. Fénérive ( $7 / 121911)$, plusieurs ex., max. long. $53 \mathrm{~mm}$. - Fandrarazana, 2 ex., max. long. $30 \mathrm{~mm}$ (Nov. 1911). Les organes génitaux s'accordent tout à fait avec la description et la figure publiée par Simroth $1910^{2}$ (p. 597, fig. 6 A). La couleur est blanche

1 Aufzählung der Nanina-Arten Madagascars. Jahrb. d. Deutsch. Malakozool. Ges., Jahrg. 9.

2 Lissopode Nacktschnecken von Madagaskar etc. Voeltzkow, Reise in Ostafrika, Bd III, H. 5, Stuttgart. 
avec une trace de deux bandes longitudinales au dos du bouclier.

Cyclostoma bicarinatum Sowerby. Fandrarazana (Nov. 1911), 1 ex., diam. $30 \mathrm{~mm}$.

C. zonatum Petit. Mangabe (Févr. 1912), 2 ex., max. haut. $26 \mathrm{~mm}$.

Vaginula elegans Heynemann. Pl. 4, fjg. 56-58. Tamatave $(20 / 11912)$, sur un trone d'arbre à la pluie, plusieurs ex., max. long. $38 \mathrm{~mm}$. - La couleur s'accorde avec ceile décrite par Semper $1885,{ }^{1}$ et citée par Simroth $1913^{2}$ d'après Heynemann. Surtout la dernière description attire l'attention sur la réticulation caractéristique qui se forme de manière que le ton de couleur, "der zu beiden Seiten der Mittellinie dichter wird, schnörkelartige Figürchen bildet und sich in einiger Entfernung von den Mantelkanten zu einer schwarzen Zeichnung verdichtet, die aus einer unregelmässigen Linie besteht, von welcher aus nach oben und unten kreisförmig gebogene Schnörkelchen abzweigen». A cette description se rapporte le mieux la figure 21, pl. XXVII, de Semper (l. c.).

Quant aux caractères extérieurs de cette espèce on doit signaler la forme de la sole du pied qui a une largeur uniforme et l'extrémité postérieure largement arrondie (Pl. 4, fig. 58).

L'anatomie intérieure de $V$. elegans s'accorde dans les cas où elle a été étudiée par SEMPER aux conditions prévalant chez les exemplaires présents. Cependant, le pénis (fig. 3) n'est pas "drehrund", comme Semper le marque, mais un peu aplati et presque linguiforme, à section ovale; le vas deferens déhouche à l'extrémité antérieure arrondie du pénis. Un peu au-dessus de sa base le pénis est ceint d'une enflure en forme d'anneu; au-dessous de celle-ci son pied est renflé et, tout à la base, passe dans la gaine extérieure, à l'intérieur de laquelle il reste, par ce fait, librement renfermé. La glande péniale monte à la demi-longueur du pénis, mais à une largeur double, et porte 13 sacs coecaux égalant le pénis en longueur.

1 Landmollusken, H. VII, Reisen im Archipel der Philippinen 11: 3. Wiesbaden.

${ }^{2}$ Úber die ... a auf Madagaskar etc. erbeuteten Vaginuliden etc. Voeltz kow, Reise in Ostafrika, Bd III, H. 3, Stuttgart. 
Les nerfs pédieux sortent des ganglions pédieux, comme le dit Semper, tout à fait séparés.

La glande pédieuse, qui n'est pas mentionnée par SEmper, est réfléchie et un peu repliée. Elle est donc du même type que chez le groupe Eleutherocaulis Siмrotн et tout à fait différente de la glande directe chez le genre Desmocaulis Simroth. A ce genre Simroth réfère la $V$. seychellensis Fischer, avec laquelle il identifie la $V$. elegans Heynemann. Mais cette identification se rapporte à l'autre exemplaire de Semper, figuré à la pl. XXV, fig. 12 , et non pas à celui qui s'accorde arec la description orginale de Heynemann, et

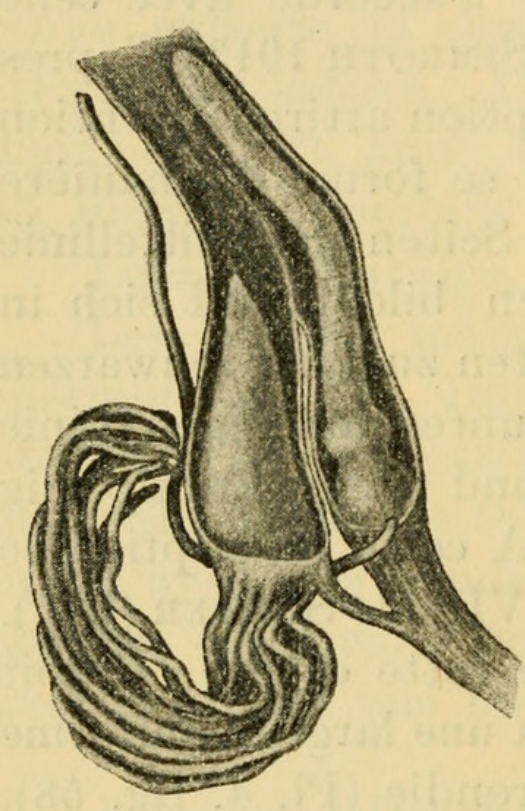

Fig. 3.

Appareil génital mâle de Vaginula elegans. auquel ressemblent les exemplaires présents. De ce dernier exemplaire Simroth constate (l. c., p. 139), 》dass die Figuren von der Schnecke von Mauritius samt der Beschreibung ihrer Anatomie nicht auf die $V$. seychellensis passen». Cette forme est, cependant, avec grande certitude la forme typique, et l'autre, qui a probablement été déterminée à faux par HeynemanN (Semper dit que ses exemplaires furent déterminés par HeYnemanN) est probablement identique à $V$. seychellensis. Par conséquent, $V$. elegans n'est pas à considérer comme synonyme de $V$. seychellensis; au contraire elle doit être établie comme type d'un groupe distinct appartenant au genre Eleutherocaulis (à cause de la forme de la glande pédieuse). Dans ce genre l'espèce présente occupe une place coordonnée à et séparée de Lavicaulis ainsi que d'Annulicaulis, desquelles le premier sous-genre possède un pénis cylindrique et lisse jusqu'à l'extrémité, tandis que le pénis du dernier est pourvu d'un anneau à sa pointe. L'espèce en question forme, à cause de son anneau basal, un sousgenre distinct, qui peut être dénommé Vallicaulis et qui rappelle le genre Vaginina (voir Simroth, l. c., p. 193).

V. ocellata n. sp. Pl. 4, fig. 59, 60. Corps allongé-elliptique, un peu plus étroit à l'extrémité postérieure qu'à l'antérieure. Couleur (en alcool) sur le dos bleue-grise foncée (le 
tiers antérieur gris-clair) et avec des taches blanchâtres plus ou moins grandes avec ou sans un cercle foncé. Le long de la moitié postérieure du dos une ligne jaune-claire. La surface inférieure a un périnotum gris-foncé; tentacules supérieurs gris-bruns foncés, les inférieurs gris-clairs; la sole du pied d'une couleur uniformement grise-claire. Longueur 48, largeur 18 , hauteur $14 \mathrm{~mm}$.

L'ouverture femelle immédiatement derrière la ligne en travers (plus approchée de celle-ci que chez $V$. elegans). Anus

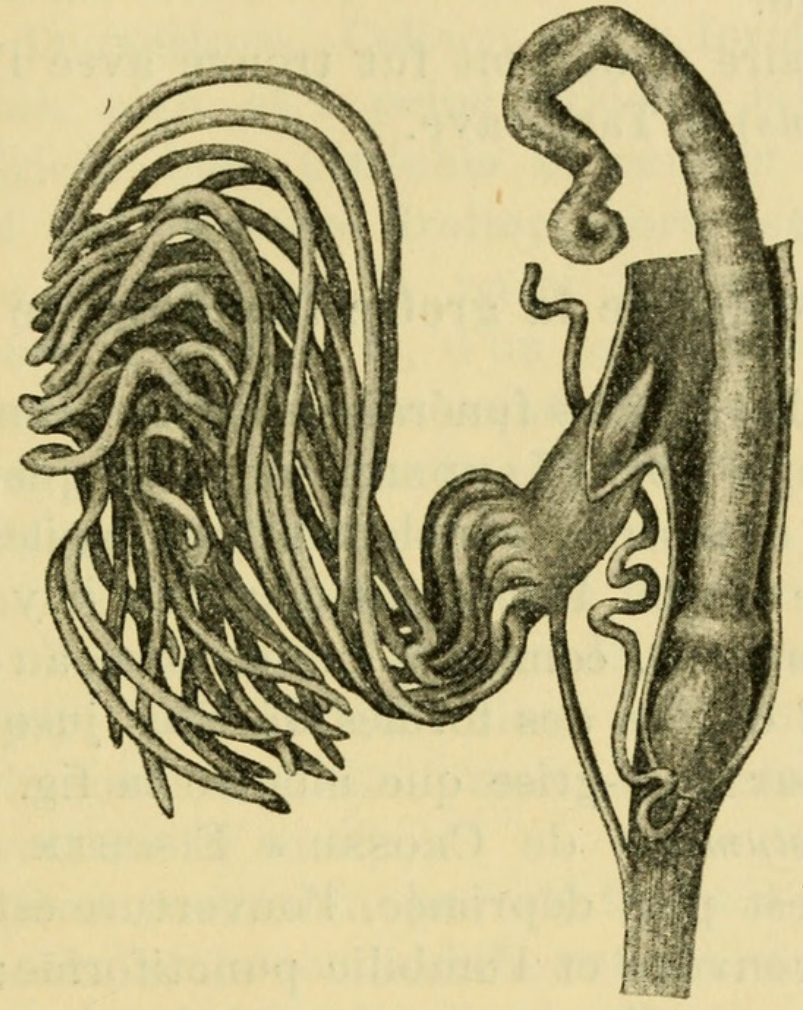

Fig. 4.

Appareil génital mâle de Vaginula ocellata.

au côté de la ligne médiane dans l'extrémité postérieure du corps. Périnotum égalant la sole en largeur. Celle-ci un peu plus étroite en arrière que chez $V$. elegans.

L'anatomie intérieure montre un pénis (fig. 4) très allongé pourvu immédiatement au-dessus de sa base d'un renflement en forme d'anneau, au-dessus de celui-là rond, vers la pointe presque trigonal, la crête en dehors, puis réfléchi et après la flexion avec une partie courte aplatie et linguiforme, terminant en un processus digitiforme enroulé.

Glande péniale conique allongée, considérablement plus courte que la demi-longueur du pénis (sa longueur égalant à peu près de la base du pénis à l'anneau), et un peu plus 
étroite que le pénis au-dessus de l'anneau. Vesicules muqueuses 18, simples, cordiformes, leur longueur deux fois celle du pénis étendu et presque égale à la demi-longueur de l'animal.

Les nerfs pédieux sortent bien séparées des ganglions pédieux.

La glande pédieuse est réfléchie et un peu repliée.

L'espèce présente qui est nettement distincte de $V$. elegans appartient au même groupe (Vallicaulis) du genre Eleutherocaulis que celle-ci à cause du renflement annulaire à la base de la verge.

Un exemplaire seulement fut trouvé avec l'espèce précédente $(V$. elegans) à Tamatave.

\section{Mollusques de la grotte funéraire de Catsèpe.}

Dans une des grottes funéraires aux environs de Catsèpe, près de Majunga, Dr. KAUDERN trouvait quelques espèces de mollusques terrestres, visiblement subfossiles et couverts d'une croûte de terre. Parmi ces coquilles il y a une petite espèce qui appartient, comme il me semble, au genre Microcystis, mais qui diffère des formes décrites jusqu'ici. Elle a la mème couleur rose-grise que montre la fig. 5 (pl. 19) de Macrochlamys stumpfii de Crosse \& Fischer (1889), ${ }^{1}$ mais toute sa taille est plus déprimée, l'ouverture est plus étroite, la base moins convexe et l'ombilic punctiforme; diam. de la coquille 6,8 mm. - Il se trouve aussi des fragments de coquilles d'une largeur assez grande; entre eux il y a un morceau à 3 bandes brunes alternant de blanches (peut-être appartenant à Ampelita atropos Fér.). Une quantité de fragments présentent un morceau du dernier tour, y compris l'ombilic, qui est très étroit, d'une coquille fragile dont la couleur est uniformement blanche à la base, tirant dans le jaune vers la suture, où la surface est lisse, tandis qu'elle est très finement striée en spiral à la base de la coquille. Un petit morceau d'un des tours supérieurs, sans doute de la même espèce, montre la couleur jaunâtre formant deux bandes, alternant avec trois zones brunes. Les fragments semblent appartenir au genre Nanina.

1 Hist. physique, naturelle et politique de Madagascar. Vol. XXV. Hist. nat. des Mollusques, Paris. 
Il y a aussi quelques espèces qui sont mieux déterminables; ce sont les suivantes, sur lesquelles trois sont nouvelles pour la science.

Ampelita bizonalis n. sp. Pl. 4, fig. 53-55. Coquille très déprimée, largement et perspectivement ombiliquée; surface un peu luisante et sculptée de stries d'accroissement bien marquées, irrégulières, et microscopiquement rugueuse entre elles. Tours 5, aplatis, séparés d'une suture fine, le dernier presque anguleux à la périphérie, assez élargi et brusquement descendant à l'ouverture. Celle-ci d'une forme ovale, à labre supérieur droit, aigu, et à peine réfléchi; labre extérieur et surtout l'inférieur bien réfléchis et ceux-ci sinueux vers la columelle qui est presque droite; labres continus par une mince couche de cal sur la paroi interne. Couleur d'un brun foncé vers la périphérie, d'un jaune-brun plus clair aux tours supérieurs; la suture marquée d'une ligne blanchâtre; à l'angle du dernier tour une bande étroite de fauve et une autre, plus large, entourant l'ombilic, qui est, en dedans, coloré du même brun foncé que le dernier tour; la partie inférieure de l'ouverture d'une couleur chocolat avec une couche de blanc, labre réfléchi blanc inférieurement, le reste brunâtre. Dimensions: Diam. max. 36 ; min. 28; haut. 17; larg. de l'ouverture $18 \mathrm{~mm}$.

Cette espèce ressemble par sa forme à. l'Ampelita lanx ou à l'A. lancula, mais son ombilic est plus ample, la coquille est plus déprimée et plus fragile, le dernier tour plus élargi vers l'ouverture. Comme je n'ai pas retrouvé cette forme dans les monographies illustrées, je pense qu'il est justifié de la signaler comme nouvelle espèce.

Cyclostoma ligatum MüLler. 3 ex., max. haut. $13 \mathrm{~mm} .-$ Comparés avec des exemplaires typiques de Port Natal, ils montrent tout à fait les caractères de cette espèce: une coquille largement ombiliquée à costules spirales autour de l'ombilic et de semblables un peu plus faibles sur le reste de la surface.

La couleur est d'un gris-jaune pâle avec une bande brune étroite au-dessous du milieu du dernier tour et quelques-unes plus fines au-dessus d'elle. L'espèce, qui se trouve à la côte orientale de l'Afrique n'a pas été signalée de Madagascar.

Arkiv för zoologi. Band 12. N:o 6. 
Cyclotus mamillaris n. sp. Pl. 4, fig. 48, 49. Coquille discoïde, déprimée, à spire papilliforme, et à $3 \frac{1}{1} / 2$ tours convexes, très largement et perspectivement ombiliquée, d'une couleur blanchâtre et d'une surface luisante, sculptée de costules spirales découpées par des côtes minces transversales, régulièrement espacées, plus visibles vers l'ouverture; les deux tours formant le sommet lisse. Ouverture circulaire, péristome simple, continu. Opercule inconnu. Dimensions: Max. diam. 3,3 ; min. diam. 2,8 ; haut. $1 \mathrm{~mm}$.

Cette petite coquille, par sa grandeur peu considérable et sa forme discoïde, ressemble au Cyclotus ispingoënsis STURANY (de Port Natal), mais elle diffère de cette espèce par sa spire papilliforme et par sa sculpture; aussi le dernier tour ne descend pas à l'ouverture comme chez cette espèce. Le genre reste incertain, l'opercule manquant.

Omphalotropis aurata n. sp. Pl. 4, fig. 46, 47. Coquille rissoïde, à spire élevée et à tours bien convexes, le dernier un peu renflé; suture profonde. Ombilic en forme de sillon trés étroit, entouré d'une carène très mince. Overture assez oblique, lunate, plus courte que la demi-hauteur de la coquille; péristome simple; columelle un peu élargie et aplatie, à bord droit pourvu d'une petite convexité; une mince couche de cal s'étend sur la paroi interne. Couleur orange, columelle blanche. Sculpture composée de stries spirales très fines et serrées, croisées de stries d'accroissement; sommet lisse. Tours 4. Dimensions: hauteur 2,7, largeur 2, haut. de l'ouverture $1 \mathrm{~mm}$.

Cette coquille semble appartenir au sous-genre Eurytropis Kobelt \& MoellendorfF. Sa petitesse et le petit nombre de ses tours indiquent qu'elle est, probablement, un jeun individu. Comme elle ne s'accorde à aucune description des espèces connues de ce genre, et n'a pas été rapportée jusqu'ici connue de Madagascar, j'ai décidé de la signaler comme forme nouvelle. 


\section{Explication des planches.}

\section{PI. 1.}

1. Pinna bicolor Chemnitz, var. Mahakamby. ca. ${ }^{2 / 3}$.

2, 3. Diplodonta subcostata $\mathrm{n}$. sp., valve droite. Majunga. $\times 2,8$.

4. Scintilla symmetrica n. sp., valve droite. Majunga. $\times 2,8$.

5-8. Donax elegans n. sp., 2 ex. Majunga. $\times 1,7$.

9. Pyramidella (Elusa) subglabra n. sp. Majunga. $\times 4,5$.

10-12. Siphonaria madagascariensis n. sp. Majunga, 2 ex., l'un de l'extérieur (fig. 10, 11), l'autre de l'intérieur. $\times 3$.

\section{PI. 2.}

13, 14. Yoldia divaricata n. sp. Tamatave. $\times 2,6$.

15-18. Nucula rugosa $\mathrm{n}$. sp. Tamatave. $\times 2,6$.

19. Lucina elongata $\mathrm{n}$. sp. Tamatave. $\times 2,8$.

20, 21. Syndesmya (Iacra) lactea Dunker. Tamatave. $\times 2,5$.

22, 23. Donax productus n. sp. Tamatave. $\times 2,5$.

24. ?Cultellus attenuatus Dunker. Tamatave. $\times$. 4 .

25. Phasianella zigzag n. sp. Fénérive. $\times 9$.

26-28. Minolia variegata $n$. sp. Tamatave. $\times 2$, .

\section{Pl. 3.}

29. Mitra (Turricula) interpunctata n. sp. Tamatave. $\times 2,5$.

30. Terebra polygonia Reeve. Tamatave. $\times 2,5$.

31. Cirsotrema soror n. sp. Tamatave. $\times 2,5$.

$32-33$. C. soror, sculpture $\times 8$ (fig. 32) et $\times 12$ (fig. 33 ).

34. Ovula insculpta n. sp. Majunga. $\times 2,5$.

35-39. Chama spinosa BRoderip. Tamatave. $\times 1,8$.

40. Ischnochiton rufopunctatus $\mathrm{n}$. sp. Majunga. $\times 6$.

41. I. rufopunctatus, sculpture d'une valve intermédiaire, demie gauche. $\times 20$. 
42. Acanthochites aberrans n. sp. Majunga. $\times 6$.

43. A. aberrans, sculpture d'une valve intermédiaire. $\times 27$. 44-45. Choneplax indicus n. sp. Tamatave. $\times 4,5$.

\section{Pl. 4.}

46-47. Omphalotropis aurata $\mathrm{n}$. sp. Catsèpe, grotte funéraire. $\times 8$. 48-49. Cyclotus mamillaris n. sp. 》 $》 \gg \times 8$. 50-52. Nanina hova n. sp. 》 》 " 53-55. Ampelita bizonalis n. sp. 》 》 》 56-58. Vaginula (Vallicaulis) elegans Heynemann. Tamatave. 》 59-60. V. (Vallicaulis) ocellata n. sp. 


\section{$2 \mathrm{BHL}$ Biodiversity Heritage Library}

Odhner, Nils Hj. 1919. "Contribution à la faune malacologique de Madagascar." Arkiv för zoologi 12, 1-52. https://doi.org/10.5962/bhl.part.789.

View This Item Online: https://www.biodiversitylibrary.org/item/30230

DOI: https://doi.org/10.5962/bhl.part.789

Permalink: https://www.biodiversitylibrary.org/partpdf/789

\section{Holding Institution}

MBLWHOI Library

\section{Sponsored by}

MBLWHOI Library

\section{Copyright \& Reuse}

Copyright Status: NOT_IN_COPYRIGHT

This document was created from content at the Biodiversity Heritage Library, the world's largest open access digital library for biodiversity literature and archives. Visit BHL at https://www.biodiversitylibrary.org. 